\title{
A Two-Way Interaction between Methotrexate and the Gut Microbiota of Male Sprague-Dawley Rats
}

Marine P. M. Letertre,* Nyasha Munjoma, Kate Wolfer, Alexandros Pechlivanis, Julie A. K. McDonald, Rhiannon N. Hardwick, Nathan J. Cherrington, Muireann Coen, Jeremy K. Nicholson, Lesley Hoyles, Jonathan R. Swann, and Ian D. Wilson*

Cite This: https://dx.doi.org/10.1021/acs.jproteome.0c00230

S1 Supporting Information

ABSTRACT: Methotrexate (MTX) is a chemotherapeutic agent that can cause a range of toxic side effects including gastrointestinal damage, hepatotoxicity, myelosuppression, and nephrotoxicity and has potentially complex interactions with the gut microbiome. Following untargeted UPLCqtof-MS analysis of urine and fecal samples from male Sprague-Dawley rats administered at either $0,10,40$, or $100 \mathrm{mg} / \mathrm{kg}$ of MTX, dose-dependent changes in the endogenous metabolite profiles were detected. Semiquantitative targeted UPLC-MS detected MTX excreted in urine as well as MTX and two metabolites, 2,4-diamino- $N$-10-methylpteroic acid (DAMPA) and 7-hydroxyMTX, in the feces. DAMPA is produced by the bacterial enzyme carboxypeptidase glutamate 2 (CPDG2) in the gut. Microbiota profiling (16S rRNA gene amplicon sequencing) of fecal samples showed an increase in

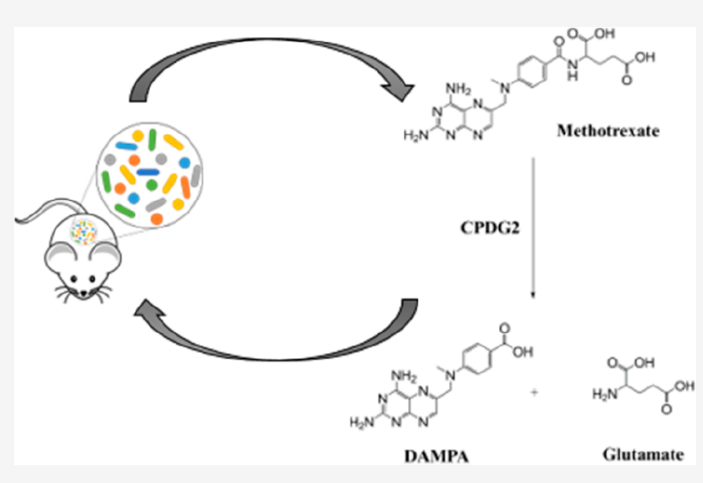
the relative abundance of Firmicutes over the Bacteroidetes at low doses of MTX but the reverse at high doses. Firmicutes relative abundance was positively correlated with DAMPA excretion in feces at 48 h, which were both lower at $100 \mathrm{mg} / \mathrm{kg}$ compared to that seen at $40 \mathrm{mg} / \mathrm{kg}$. Overall, chronic exposure to MTX appears to induce community and functionality changes in the intestinal microbiota, inducing downstream perturbations in CPDG2 activity, and thus may delay MTX detoxication to DAMPA. This reduction in metabolic clearance might be associated with increased gastrointestinal toxicity.

KEYWORDS: methotrexate, gastrointestinal toxicity, gut microbiome, metabolic phenotyping, mass spectrometry, amplicon sequencing

\section{INTRODUCTION}

Methotrexate (MTX, amethopterin, 4-amino-4-deoxy- $N$-10methylpteroylglutamic acid) is a folate analogue used to treat a range of different pathologies. At doses of $<15 \mathrm{mg} / \mathrm{kg} \mathrm{MTX}$ is widely used to treat autoimmune diseases such as rheumatoid arthritis (RA) or psoriasis. At higher doses, usually between 15 and $1000 \mathrm{mg} / \mathrm{kg}$, the drug is effective in the treatment of neoplastic diseases, mainly for acute lymphocytic leukemia, lymphoma, and osteosarcoma. ${ }^{2}$ At low doses, MTX shows antiinflammatory properties, but with prolonged use, or with high doses, it can induce nephrotoxicity, life-threatening myelosuppression, gastrointestinal toxicity and hepatotoxicity. ${ }^{3} \mathrm{MTX}$ is metabolized in the liver by aldehyde oxidase (AO) into 7 hydroxy-MTX (7-OH-MTX), and both the parent compound and the metabolite are transported into cells by carrier proteins. ${ }^{4,5}$ These metabolites can undergo polyglutamation, and the products of this reaction inhibit dihydrofolate reductase (DHFR) and influence folate metabolism. ${ }^{6}$ This inhibition blocks tetrahydrofolate (THF) production from folic acid, inhibiting purine and pyrimidine synthesis and downstream DNA synthesis.?
The intestinal microbiota are increasingly recognized as participating in the processing of many xenobiotics and can also influence host responses to numerous compounds. ${ }^{8-12}$ However, our understanding of these processes remains limited. The gut microbiota can directly participate in drug metabolism, as occurs with MTX. Thus, following absorption and hepatic metabolism of MTX to generate 7-OH-MTX, these molecules are excreted into the bile and subsequently into the gastrointestinal tract. Here, they can undergo further biotransformation by the gut microbiota. ${ }^{13,14}$ A bacterial enzyme, carboxypeptidase glutamate 2 (CPDG2; also referred to as folylpolyglutamate carboxypeptidase [FGCP]), can cleave the terminal glutamate residues of MTX and 7-OH-MTX ${ }^{14,15}$ resulting in metabolites such as 2,4-diamino- $\mathrm{N}$-10-methyl-

Received: April 6, 2020

Published: June 16, 2020 
pteroic acid (DAMPA) and 7-hydroxy-DAMPA, respectively. ${ }^{16}$ In addition, bacterial enzymes such as $p$-aminobenzoylglutamate hydrolase, found in $E$. coli, have also been shown to catalyze this reaction. ${ }^{17}$ As these produced metabolites do not have activity toward DHFR inhibition, glutamate hydrolysis by CPDG2 is seen as a detoxication process. ${ }^{18,19}$ As such, CPDG2 is approved as a rescue agent in cancer patients presenting delayed MTX clearance and acute nephrotoxicity, ${ }^{2}$ and several clinical studies have reported positive results for its use in this role. ${ }^{3,20,21}$ Even so, MTX and its relatively insoluble metabolites are still presumed to precipitate in kidneys, causing nephrotoxicity, ${ }^{3}$ as well as causing severe gastrointestinal toxicity such as vomiting, diarrhea, oral mucositis and, in some cases, the death of patients. $^{22}$

Therapeutic treatment can also impact the community structure of the gut microbiota modulating its diversity and functionality. MTX has recently been reported to inhibit the growth of $30 \%$ of 40 representative gut bacterial strains ${ }^{23}$ and $84 \%$ of 43 bacterial isolates which had a combined relative abundance covering $43 \%$ of the human gut microbiota. ${ }^{24}$ Studies exploring the effects of MTX on the gut microbiota have previously focused on the anti-inflammatory role of MTX in RA. Low doses (i.e., at or below $15 \mathrm{mg} /$ week) to patients increased the species richness and diversity of the microbiota and reversed the perturbation of the microbiota that is often associated with RA. ${ }^{25,26}$ In a recent study, ${ }^{24}$ MTX treatment (10 or $50 \mathrm{mg} / \mathrm{kg}$ ) of gut microflora-humanized gnotobiotic mice decreased the relative abundance of Bacteroidetes and increased that of the Firmicutes. As the initial targeted human enzyme DHFR is conserved in all domains of life, such alterations were further demonstrated as an anti-inflammatory mechanism of MTX through potential interaction with offtarget bacterial enzymes, such as DHFR in Escherichia coli or Lactobacillus casei. ${ }^{27}$ However, higher doses of MTX (90 mg/ $\mathrm{kg}$ i.p.) administrated to rats with conventional microbiota caused mucositis and a reduction, by 705 -fold, of bacterial diversity, compared to untreated control rats. ${ }^{28}$ Using a cell enumeration method (flow-FISH), the majority of the alterations were seen to have occurred via a relative decrease in anaerobes belonging to the Firmicutes, accompanied by a relative increase in Bacteroides. This was in contrast to the study performed in humanized gnotobiotic mice, which showed a decrease in abundance of the phylum Bacteroidetes. $^{24}$ In a rat model, the abundance of Bacteroides increased by $49 \%$, while a decrease (by $58 \%$ ) was seen in a range of bacteria including Clostridium, Ruminococcus, Eubacterium, and Bifidobacterium. ${ }^{28}$ By perturbing the gut microbiota, high doses of MTX may modify the abundance, or functionality, of bacterial enzymes responsible for MTX hydrolysis, such as CPDG2, which could result in downstream consequences for the inactivation/detoxication of MTX into DAMPA. Initial CPDG2 concentrations in such a bidirectional interaction could perhaps explain differences in patient therapeutic responses to MTX, and also toxicities. As such, a dose-dependent two-way interaction is proposed between MTX and the gut microbiota.

To further explore the bidirectional interaction between MTX and the gut microbiota, the impact of drug-free dose vehicle and three different doses of MTX $(10,40$, and $100 \mathrm{mg} /$ $\mathrm{kg}$ ) were compared in male Sprague-Dawley rats. The fecal microbiota was characterized using 16S rRNA gene amplicon sequencing, while the urinary and fecal metabolic phenotypes were measured using a UPLC-MS-based metabolomic approach. In addition, the metabolism of MTX itself was investigated using a semiquantitative UPLC-MS assay.

\section{MATERIALS AND METHODS}

\section{Chemical and Reagents}

For sample preparation and UPLC-MS, LC-MS Chromasolv acetonitrile $(\mathrm{ACN})$ and methanol $(\mathrm{MeOH})$ were purchased from Honeywell (Seelze, Germany), Optima LC/MS grade water with $0.1 \%$ formic acid (FA) and analytical reagent grade FA were from Fisher Scientific Ltd. (Loughborough, UK). For UPLC-MS mass accuracy and calibration, leucine enkephalin acetate salt (hydrate) and sodium formate, as well as sodium azide for fecal sample preparation were obtained from SigmaAldrich (Gillingham, U.K.). The chemical standards, MTX, DAMPA, 7-OH-MTX were obtained from Toronto Chemicals (Toronto, Canada).

\section{Study Design}

As reported in detail elsewhere, ${ }^{29}$ the animal study was performed at the University of Arizona and was approved by the Institutional Animal Care and Use Committee (IACUC) and in accordance with NIH guidelines. Male SpragueDawley rats $(N=22), 7$ to 8 weeks old, weighing between 200 and $250 \mathrm{~g}$ (Harlan Laboratories, Indianapolis, IN) were fed ad libitum with a control diet (choline sufficient and amino aciddefined) (Dyets, Bethlehem, PA) and separated into four MTX dose-related subgroups $(0,10,40$, and $100 \mathrm{mg} / \mathrm{kg}$, i.p., dissolved in $0.3 \mathrm{M}$ sodium bicarbonate). The animals were randomly placed, $12 \mathrm{~h}$ prior to MTX administration, into metabolism cages. Urine samples were collected between -6 and $0 \mathrm{~h}$ (predose, $0 \mathrm{~h}), 6$ and $12 \mathrm{~h}(12 \mathrm{~h}), 18$ and $24 \mathrm{~h}(24 \mathrm{~h})$, and between 36 and $48 \mathrm{~h}(48 \mathrm{~h})$; a total of 5 samples per time point were collected. Fecal samples were collected between $-12 \mathrm{~h}$ and $-6 \mathrm{~h}$ and between -6 and $0 \mathrm{~h}$ and combined for analysis (predose, $0 \mathrm{~h}$ ), between 6 and $12 \mathrm{~h}(12 \mathrm{~h})$ and between $36-48 \mathrm{~h}(48 \mathrm{~h})$ postdose. Due to diarrhea in some MTX-treated rats, it was not possible to collect all of the postdose fecal samples (see the Results section for details).

\section{Fecal Water Preparation for UPLC-MS Analysis}

Fecal samples were defrosted and $50 \mathrm{mg}( \pm 1.4 \mathrm{mg})$ were weighed for the first biological replicate and $51 \mathrm{mg}( \pm 1.3 \mathrm{mg})$ for the second. Samples were mixed with $1 \mathrm{~mm}$ diameter zirconium beads (Stratech Scientific Ltd., Ely, UK) and $1.1 \mathrm{~mL}$ of $\mathrm{H}_{2} \mathrm{O}$ (3.3 $\mathrm{mM} \mathrm{NaN}$ ) before being vortexed and homogenized with a Precellys 24 bead beater (40 s per cycle, $6500 \mathrm{~Hz}$ speed, 2 cycles). After 20 min of centrifugation ( $17000 \mathrm{~g}$ ), the supernatants were collected and $1.1 \mathrm{~mL}$ of $\mathrm{H}_{2} \mathrm{O}$ (3.3 $\mathrm{mM} \mathrm{NaN}_{3}$ ) was added to the residue, followed by an additional step of bead beating in $\mathrm{H}_{2} \mathrm{O}\left(3.3 \mathrm{mM} \mathrm{NaN}_{3}\right)$ and centrifugation to optimize metabolite extraction. The two supernatants were combined and $250 \mu \mathrm{L}$ of the combined fecal extract were transferred to a 96-well plate. For quality control (QC) purposes, QC samples were prepared by mixing $150 \mu \mathrm{L}$ of each sample from the animals which had not received any MTX to provide a pooled sample ${ }^{30,31}$ from which $250 \mu \mathrm{L}$ were also placed into the 96-well plate. The samples were concentrated under a nitrogen flow for $3 \mathrm{~h}$. Then $120 \mu \mathrm{L}$ of $\mathrm{MeOH}: \mathrm{H}_{2} \mathrm{O}$ (1:1) were added to each sample and the plates were centrifuged ( $5 \mathrm{~min}, 700 \mathrm{~g}$ ). For each centrifuged sample $100 \mu \mathrm{L}$ were diluted with $100 \mu \mathrm{L}$ of acidified $\mathrm{H}_{2} \mathrm{O}$ (0.1\% FA) 
and transferred, in a randomized order, into 96-well plates and placed into the LC-MS autosampler at $4{ }^{\circ} \mathrm{C}$.

\section{Urine Sample Preparation for UPLC-MS}

Urine samples were prepared as described previously. ${ }^{32}$ Briefly, $20 \mu \mathrm{L}$ of urine were mixed with $60 \mu \mathrm{L}$ of $\mathrm{MeOH}$ and stored at $-20{ }^{\circ} \mathrm{C}$ overnight for protein removal. Samples were centrifuged ( $5 \mathrm{~min}, 15,000 \mathrm{~g}, 4^{\circ} \mathrm{C}$ ) and $25 \mu \mathrm{L}$ of supernatant were transferred into a 96-well plate in a randomized order. For a QC sample a $30 \mu \mathrm{L}$ aliquot of each control sample was pooled. A volume of $225 \mu \mathrm{L}$ of water was added to each sample and the plates were centrifuged $(5 \mathrm{~min}, 700 \mathrm{~g})$ before transfer to the autosampler at $4{ }^{\circ} \mathrm{C}$.

\section{UPLC-MS}

Analysis was performed as previously described ${ }^{33}$ on a Waters Acquity I-class UPLC system (Waters Corp., Milford, MA) with separation on a HSS T3 $1.8 \mu \mathrm{M}$ column $(2.1 \mathrm{~mm}$ i.d. $\times$ $150 \mathrm{~mm}$ ). The column temperature used was $45{ }^{\circ} \mathrm{C}$ and the autosampler temperature was set at $4{ }^{\circ} \mathrm{C}$. The volume of sample injected was $5 \mu \mathrm{L}$. Mobile phases were water with $0.1 \%$ FA (v/v) (solvent A) and ACN with $0.1 \%$ FA (v/v) (solvent $\mathrm{B})$, using a flow rate of $0.6 \mathrm{~mL} / \mathrm{min}$. The gradient began at 99\% A, and mobile phase B increased from 1 to $55 \%$ between $0.10 \mathrm{~s}$ and $10 \mathrm{~min}$. Between 10 and $10.65 \mathrm{~min}$ mobile phase $\mathrm{B}$ increased to $100 \%$ and the flow rate was increased to $0.8 \mathrm{~mL} /$ min, holding for $1 \mathrm{~min}$ as a wash step, followed by reequilibration of $1 \mathrm{~min}$ at $99 \% \mathrm{~A}$ (total run time: $12.65 \mathrm{~min}$ ). Prior to sample analysis, the column was conditioned with 20 QCs, ${ }^{30,31}$ and a QC was then injected every 11 samples to enable the reproducibility and consistency of the analysis to be monitored.

Mass spectrometry was performed using a Synapt G2-S mass spectrometer (Waters Corp., Wilmslow, U.K) using electrospray ionization in positive mode (ESI+). The capillary voltage was $1.5 \mathrm{kV}$ and the source temperature was set to $120^{\circ} \mathrm{C}$. The cone gas flow was $50 \mathrm{~L} / \mathrm{h}$ and the gas used was nitrogen. The desolvation gas temperature was $450{ }^{\circ} \mathrm{C}$ at a flow rate of 900 $\mathrm{L} / \mathrm{h}$. The nebulizer gas flow was 6 bar. Data acquisition was carried out over the $\mathrm{m} / z$ range 50-1200 and data were collected in centroid mode with a collision energy ramp of 15 to $45 \mathrm{eV}$. Leucine encephalin $(\mathrm{MW}=556.27 \mathrm{Da})$ was used to monitor mass accuracy with a scan collected every $60 \mathrm{~s}$ and a cone voltage of $30 \mathrm{~V}$. The data were collected using MassLynx V 4.1 (Waters Corp., Manchester, U.K.). In order to perform semitargeted analysis for MTX and its related metabolites, calibration curves for MTX, DAMPA and 7-OH-MTX were prepared by addition of the analytes to the fecal water $(0.05$, $0.5,2,8,10,16,20 \mu \mathrm{g} / \mathrm{mL})$ and urine matrices $(0.1,2,8,10$, $16,20,30 \mu \mathrm{g} / \mathrm{mL})$. Run order and batch effects were assessed using the QC data.

\section{Fecal DNA Extraction for Amplicon Sequencing}

DNA extraction was carried out on fecal samples (240 $\mathrm{mg}[ \pm$ $27 \mathrm{mg}]$ ) using the Qiagen QIAamp PowerFecal DNA kit (Mo Bio, Carlsbad, CA, USA) according to the manufacturer's protocol, with the exception that the homogenization of the samples was achieved using a Bullet Blender Strom (speed 8, 3 min) (Chembio Ltd., St Albans, UK). The Qubit dsDNA BR assay kit (Life Technologies Ltd., Paisley, UK) was used to quantify the extracted DNA. Dilution of each sample was performed to obtain a final concentration of $5 \mathrm{ng} / \mathrm{mL}$. Amplification of the V1 and V2 regions of the 16S rRNA gene was performed using the primers $341 \mathrm{~F}$ (forward) and
805R (reverse), as previously reported. ${ }^{34}$ Library pooling and quantification was carried out with NEBNext Library Quant Kit for Illumina (New England Biolabs, Hitchin, UK) and the pooled libraries were denatured before sequencing, which was performed on an Illumina MiSeq platform (Illumina Inc., Saffron Walden, UK) by using the MiSeq Reagent Kit v3 (Illumina) and paired-end 300 bp chemistry. This $16 \mathrm{~S}$ rRNA gene sequence data associated with this project have been deposited at DDBJ/ENA/GenBank under BioProject accession PRJNA599597.

\section{Targeted UPLC-MS Analysis}

The quantification of MTX, DAMPA and 7-OH-MTX by UPLC-MS was achieved using a linear calibration curve and a $\log$ transformation of the axis, using TargetLynx $\mathrm{V} \quad 4.2$ (Waters). The $R^{2}$ for the calibration curve of each of the standards was greater than 0.98 (Figure S1). As the fecal samples were prepared in duplicate, concentrations of each of the analytes were averaged. As the total volume of urine samples collected was recorded, it was possible to calculate the total amount of MTX excreted in the urine. However, the total weight of the fecal samples was not recorded and so the quantification of MTX and its metabolites excreted in feces are reported as the mass of the analytes $(\mu \mathrm{g})$ per $50 \mathrm{mg}$ of wet fecal sample.

\section{Untargeted UPLC-MS Analysis}

Preprocessing of the untargeted UPLC-MS analysis data was performed using the XCMS package implemented in $\mathrm{R}^{35-37}$ after converting the raw files into the .mzML format using MSConvert utility of the Proteowizard package 3.0. ${ }^{38}$ Peak picking was performed using the "centWave" method followed by peak grouping ("nearest" method). The data sets were normalized using median fold change method ${ }^{39}$ and a filter was applied to remove any features presenting a coefficient of variation higher than $20 \%$ in the QCs. Processed data were modeled in SIMCA 14.1 (Umetrics, Umea, Sweden). Both urine and fecal water data sets were mean-centered and logtransformed with an offset of 20, typically used for aqueous extracts. ${ }^{40}$ Principal component analysis (PCA) models were used to identify outliers and observe general trends in the data. The data sets were imported into Matlab R2018a for covariateadjusted projection to latent structures regression (CA-PLSR), ${ }^{41}$ and models were constructed adjusting for the animals, the cohort and the replicate cofounders. The goodness-of-fit and predictive power of each PLS-R model was assessed using the $R^{2} Y$ and $Q^{2} Y$ values. Features having a $q$-value ( $p$ value corrected by a multiple testing based on the BenjaminiHochberg method ${ }^{42}$ with a false discovery rate of 5\%) after a Monte Carlo Cross-Validation (MCCV, using 100 rounds and a partitioning of 3 ) below 0.05 were selected for metabolite identification. During data analysis time-related changes were identified in the profiles of the control animals. To remove the features that were unrelated to MTX administration an inhouse algorithm, written in R (see SI for further details) was used to compare the list the significantly changed features in each group and subtract those of the untreated group from the treated groups.

\section{Metabolite Identification}

Features selected as significant according to the CA-PLS-R models were putatively annotated using several different approaches. Initially, the $\mathrm{m} / z$ and the retention times were searched in the in-house National Phenome Centre Database 
(NPC, see acknowledgments). In addition, features were matched with online databases using CEU Mass Mediator, ${ }^{43}$ by comparing the $[\mathrm{M}+\mathrm{H}]^{+},\left[\mathrm{M}+\mathrm{H}-\mathrm{H}_{2} \mathrm{O}\right]^{+},[\mathrm{M}+\mathrm{Na}]^{+}$, or $[\mathrm{M}+\mathrm{K}]^{+}$adducts, as well as the main fragments (up to four, Tables S1 and S2). The level of confidence for each annotation are reported according the criteria used by the Metabolomics Standards Initiative, ${ }^{44}$ with the addition of a subconfidence group in the level 2 annotation. Annotation to a specific metabolite using one orthogonal parameter (e.g., $m / z$ values matching to a database) or two orthogonal parameters (e.g., $\mathrm{m} / z$ values and retention time) without spiking the corresponding authentic standard are reported as annotation level $2 b$ and $2 a$, respectively. Data-dependent acquisition (DDA) and UPLC-MS/MS experiments (using a Xevo G2 QToF [Waters Ltd., Elstree, UK] and the same chromatographic conditions as the original profiling) were performed on the features tentatively annotated by this approach to support these putative identifications. The capillary voltage was $3 \mathrm{kV}$ and the source temperature were set at $120^{\circ} \mathrm{C}$. The cone gas flow was $150 \mathrm{~L} / \mathrm{h}$ and the gas used was nitrogen. The desolvation gas temperature was $400{ }^{\circ} \mathrm{C}$, the desolvation gas flow was $1000 \mathrm{~L} / \mathrm{h}$. Selected masses were fragmented with collision energies of 10 and $30 \mathrm{eV}$, and the application of a ramped energy $(10-40 \mathrm{eV})$. Leucine encephalin was used as the lock mass compound. The $-\log _{10}(q$-value) multiplied by the sign of the Manhattan score of the CA-PLS-R models were used to build a heatmap of the metabolites annotated in this way.

\section{Data Analysis for Microbiota Profiling}

The sequence reads obtained from the fecal DNA extracts were analyzed using the Mothur package (v.1.39.5) ${ }^{45}$ based on the MiSeq SOP pipeline. ${ }^{46}$ The Silva bacterial database (v.128) was used to process the sequence alignment, and the Ribosomal Database Project (v.2014) was used to classify sequences based on the Wang approach. ${ }^{47}$ The entire data set (number of reads generated per samples reported in Table S.1) was exported from Mothur into R (3.4.0 through RStudio v. 1.1.463) where the package phyloseq $(\mathrm{v} \cdot 1.26 .1)^{48}$ was used to statistically explore the data. To assess $\beta$ diversity and taxonomic profile, normalization was based on the proportion method, which scales each count in a sample by dividing by the counts sum in this sample. ${ }^{48}$ Rarefying to the minimal depth of reads observed in the samples ( $N=4406$ reads) was performed to explore the $\alpha$ diversity. Illustrations of the taxonomic profile agglomerated at family and phylum levels were generated for each of the MTX dose groups and by comparing pre- and post-MTX treatment. $\beta$ diversity was assessed using PCoA of Bray-Curtis dissimilarity matrix and $\alpha$ diversity was assessed using the inverse Simpson index. Drugrelated effects such as diarrhea allowed only a small quantity of feces to be collected postdose, often below the amount required for DNA extraction for amplicon sequencing. As a consequence, only a small number of samples were available for some groups, thus precluding statistical analysis.

\section{Correlation Analysis}

In order to assess correlation between the excretion of DAMPA in feces, as well as the excretion of MTX in urine or fecal samples with the microbial taxonomic profile and the putatively annotated metabolites, correlation matrices were generated. Bacterial families present in more than one predose fecal sample from three animals treated with $100 \mathrm{mg} / \mathrm{kg}$ MTX were selected and correlated with the quantity of MTX excreted in urine samples at $12 \mathrm{~h}$ postdose and with the quantity of MTX and DAMPA excreted in fecal samples at 48 $\mathrm{h}$ postdose (according to the peak excretion of these compounds in each matrix). These same families were correlated with the putatively annotated metabolites, and these were also correlated with the drug and its metabolites. Spearman correlation was used, and the clustering method was based on single linkage clustering. Significance levels for the correlogram were calculated using the corr.mtest function in $\mathrm{R}$.

\section{RESULTS}

A combination of semiquantitative targeted and untargeted UPLC-MS based metabolic profiling approaches were used to assess the biotransformation of MTX and determine its wider biochemical impact on the urinary and fecal metabolomes of rats. In parallel, the fecal microbiota were profiled to characterize the bidirectional interplay between them and MTX.

Quantification of MTX and Its Metabolites 7-OH-MTX and DAMPA

The concentrations of MTX and its two main metabolites, 7OH-MTX and DAMPA, were measured in urine and feces using semiquantitative targeted UPLC-MS (Figure 1). In urine, MTX was the only analyte detected, at any of the time points available for analysis $(0-6,6-12,18-24$, and $36-48 \mathrm{~h})$, and was predominantly found in the $6-12 \mathrm{~h}$ samples. In the feces, animals receiving $10 \mathrm{mg} / \mathrm{kg}$ of MTX had largely excreted the drug by $12 \mathrm{~h}$ postdose while those receiving doses of 40 or 100 $\mathrm{mg} / \mathrm{kg}$ excreted the highest concentrations of the drug in the 36-48 h samples. DAMPA was detected in the fecal extracts over both time-periods for the animals treated with $10 \mathrm{mg} / \mathrm{kg}$ and mainly in the $36-48 \mathrm{~h}$ fecal collection for the animals receiving 40 or $100 \mathrm{mg} / \mathrm{kg}$. The fecal concentration of $7-\mathrm{OH}-$ MTX was negligible and was only detected in the 6-12 h postdose samples from animals receiving $10 \mathrm{mg} / \mathrm{kg}$ of MTX and the 36-48 h collections for the animals treated with 40 $\mathrm{mg} / \mathrm{kg}$. The fecal excretion of 7-OH-MTX was particularly low in the animals treated with $100 \mathrm{mg} / \mathrm{kg}$ of MTX. Similarly, the fecal concentrations of DAMPA were lower in the animals treated with $100 \mathrm{mg} / \mathrm{kg}$ of MTX compared to the group treated with $40 \mathrm{mg} / \mathrm{kg}$ for the $36-48 \mathrm{~h}$ postdose samples.

\section{MTX Effects on Urinary Metabolite Profiles}

Principal components analysis (PCA) models were built using the endogenous urinary metabolic profiles obtained by untargeted UPLC-MS analysis for each MTX dose to identify sources of variation (Figure S2). A modest time-related effect was observed in the control group, particularly by $48 \mathrm{~h}$ into the study. Supervised multivariate statistical analysis using covariate-adjusted projection to latent structures regression (CA-PLS-R ${ }^{41}$ ) analysis, was also performed to illuminate the endogenous metabolic response at each MTX dose (Figure S3). The resulting models both fitted the data well (as indicated by $R^{2} Y$ ) and allowed good prediction of the data (as determined by the $Q^{2} Y$ ). The resulting skyline significance representations (Figure S4) show that, the number of urinary features significantly varying with time was more important for the animals treated with the $100 \mathrm{mg} / \mathrm{kg}$ dose. As seen for the PCA of the untreated (control) animals (Figure S2), timedependent metabolic shifts occurred in the urinary metabolic profiles, a metabolic shift was also observed in the CA-PLS-R models of the untreated animals, which was associated with changes in the signal intensity of features over time (Figure 
a)

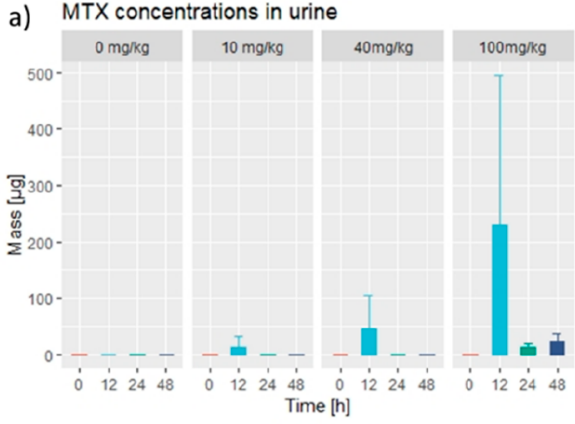

c)
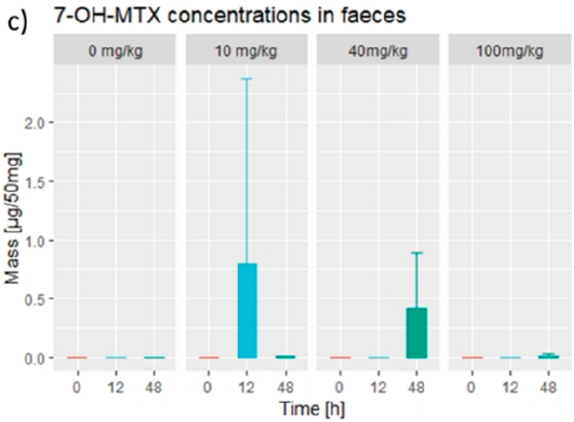

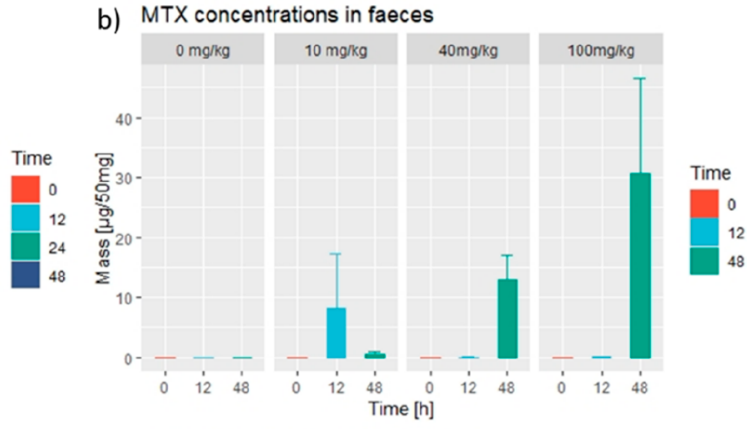

d) DAMPA concentrations in faeces

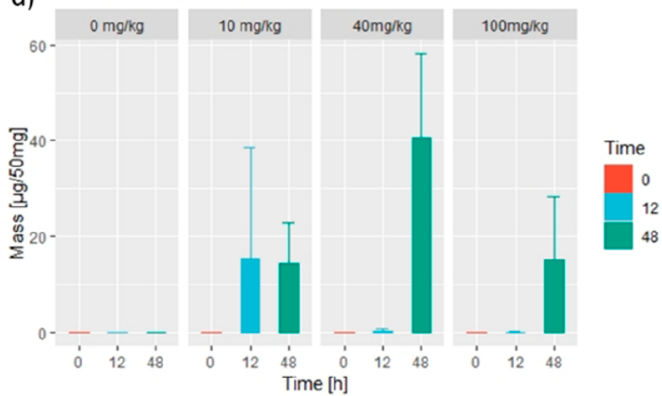

Figure 1. Quantification of MTX, 7-OH-MTX and DAMPA using UPLC-MS. (a) Excretion profile of MTX in urine, as the total amount ( $\mu \mathrm{g}$ ) in the predose $(0 \mathrm{~h}), 6$ to $12 \mathrm{~h}(12 \mathrm{~h}), 18$ to $24 \mathrm{~h}(24 \mathrm{~h})$, and 36 to $48 \mathrm{~h}(48 \mathrm{~h})$ urine collections. The fecal excretion of (b) MTX, (c) 7-OH-MTX, and (d) DAMPA, in $\mu \mathrm{g} / 50 \mathrm{mg}$ of feces from the predose $(0 \mathrm{~h}), 6-12 \mathrm{~h}(12 \mathrm{~h})$, and 36-48 h $(48 \mathrm{~h})$ collections. The bar plots represent the mean concentrations and the error bars the standard deviations. $N=5$ for each time point within each dose-related group for urine. For the fecal samples of the untreated rats, $N=6$ at $0 \mathrm{~h}, N=3$ at $12 \mathrm{~h}$, and $N=5$ at $48 \mathrm{~h}$; for the rats treated with $10 \mathrm{mg} / \mathrm{kg}, N=10$ at $0 \mathrm{~h}, N=5$ at $12 \mathrm{~h}$, and $N=4$ at 48 h; for the rats treated with $40 \mathrm{mg} / \mathrm{kg}, N=9$ at $0 \mathrm{~h}, N=3$ at $12 \mathrm{~h}$, and $N=3$ at $48 \mathrm{~h}$; and for rats treated with $100 \mathrm{mg} / \mathrm{kg}$ of MTX, N=12 at $0 \mathrm{~h}, N$ $=3$ at $12 \mathrm{~h}$, and $N=3$ at $48 \mathrm{~h}$.

S4). The features highlighted as significantly changed in the control group over time were removed from consideration when the significant features in the MTX-treated groups were examined in order to focus on, and identify, only those compounds changing as a result of drug treatment.

A large number of features (1651) were significantly altered following MTX administration. Features of interest were matched to an in-house database (based on both retention time and $m / z$ ) and to online databases (based on $m / z$ ) (Table S2). For a metabolite annotation to be reported it had to meet the criteria of having two $\mathrm{m} / z$ values matched to values from the database (obtained by $\mathrm{MS}^{\mathrm{E}}$ ) and be confirmed by MS/MS as well as biological plausibility. A heatmap based on the $-\log _{10}$ ( $q$-values) of these annotations was constructed from these data (Figure 2a). Two main clusters of metabolites emerged from this analysis, one including those that increased over time following MTX treatment and the other including those that decreased. Metabolites that decreased on dosing (following CA-PLS-Regression model with a $q$-value $<0.05$ ) included ribothymidine, pimelylcarnitine, $\mathrm{N}$-acetyl-arginine, and thymidine. Among the metabolites that increased, two subclusters can be distinguished, one includes metabolites that showed a modest increase across all MTX-dosed animals (creatine, N6-carbomoyl-threonyladenosine, $\mathrm{N}$-methyl-4-aminobenzoate, 1-methyladenosine, and xanthurenic acid), and the other included metabolites whose excretion increased in a dose-dependent manner such as acetylcarnosine, which closely clustered with methionine sulfoximine, and $\mathrm{N}$-acetyl-Lglutamine, biopterin, arginine, 5-hydroxyindoleacetic acid, creatinine, and acetylcholine.

The putative annotation of both $\mathrm{N}$-methyl-4-aminobenzoate and methionine sulfoximine is problematic as, despite good congruence with their mass spectral properties against the respective Metlin database entries, these are unlikely to have been endogenous biochemicals. For example, although methionine sulfoximine has been identified as a rare natural product found in plants of the Connaraceae family, ${ }^{49}$ it can also be found as a result of the reaction of $\mathrm{NCl}_{3}$ with proteins in wheat protein in flour treated with the chemical. ${ }^{50}$ Similarly, the putative identification of $\mathrm{N}$-methyl-4-aminobenzoate, as the most likely identity of the compound based on its MS fragmentation properties, may also have been present in the diet. However, in the case of this compound its structural similarity with that of the aromatic acid portion of DAMPA has not escaped our notice. It is possible then, that if correctly annotated, this feature may represent either a drug impurity or a novel metabolite of MTX produced via the N-dealkyation of DAMPA. The reason for the apparent differences in the relative concentrations of these two compounds in the excreta on MTX treatment may be due therefore to factors such as changes in xenobiotic metabolism or absorption, etc., rather than MTX-related effects on the endogenous metabolism of either microbiota or host.

\section{MTX Effects on the Fecal Metabolic Profile}

As was the case for urine, in the PCA models built on the fecal metabolic profiles (Figure S5), time-related variation was also observed. This occurred in the untreated $(0 \mathrm{mg} / \mathrm{kg})$ profiles along principal component one. A large amount of variation was seen in the $0-12 \mathrm{~h}$ and the $36-48 \mathrm{~h}$ samples from animals treated with MTX. This effect was dose-dependent being more pronounced at the higher doses.

CA-PLS-R models were also built to identify metabolic variation occurring over time following MTX dosing (Figure 
a)

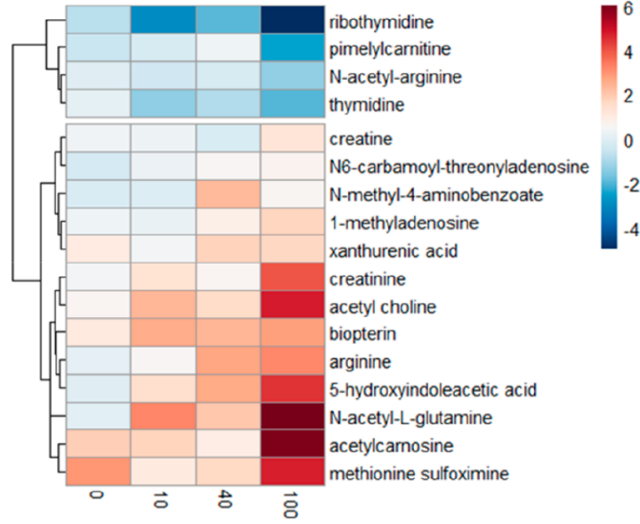

b)

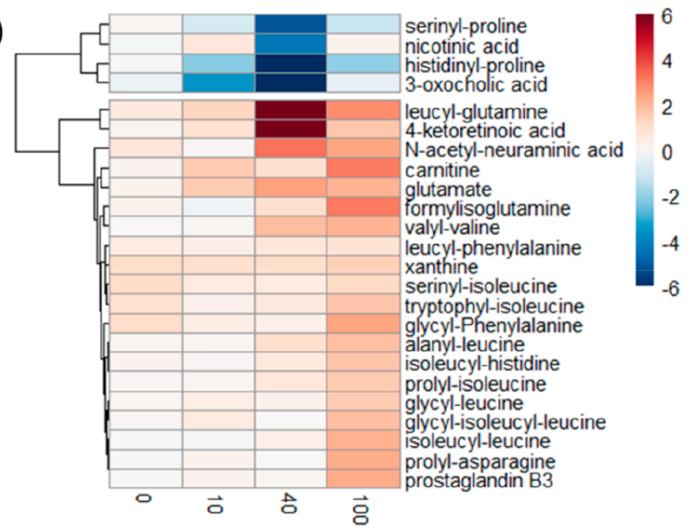

Figure 2. Heatmaps of the metabolites putatively annotated in the urine samples (a) and in the fecal samples (b), and significantly influenced by MTX treatment. Color represents the $-\log _{10}$ ( $q$-value) multiplied by the Manhattan sign of the CA-PLS-R models. Red represents an increase of the feature over time and blue indicates a decrease. $N=5$ for each time point within each dose-related group in urine. For the fecal samples of the untreated rats, $N=6$ at $0 \mathrm{~h}, N=3$ at $12 \mathrm{~h}$, and $N=5$ at $48 \mathrm{~h}$; for the rats treated with $10 \mathrm{mg} / \mathrm{kg}, N=10$ at $0 \mathrm{~h}, N=5$ at $12 \mathrm{~h}$, and $N=4$ at $48 \mathrm{~h}$; for the rats treated with $40 \mathrm{mg} / \mathrm{kg}, N=9$ at $0 \mathrm{~h}, N=3$ at $12 \mathrm{~h}$, and $N=3$ at $48 \mathrm{~h}$; and for the rats treated with $100 \mathrm{mg} / \mathrm{kg}$ of MTX, N=12 at $0 \mathrm{~h}, N=$ 3 at $12 \mathrm{~h}$, and $N=3$ at $48 \mathrm{~h}$.

S6), and, as seen for urine, these models fitted the data well and allowed good predictions to be made (based on $R^{2} Y$ and $Q^{2} Y$ ). Like urine, a number of metabolic features (644) changed significantly over time (after correction for multiple testing using the false discovery rate) for each of the MTX dose-related groups (Figure S7). The changes highlighted in the skyline significance plot of the animals treated with the 10 and $40 \mathrm{mg} / \mathrm{kg}$ doses were similar. However, a greater increase in biochemical perturbations and their intensity was observed in the skyline significance plot of the animal group treated with $100 \mathrm{mg} / \mathrm{kg}$ of MTX compared to the lower dose groups. The features significantly changing over time in the untreated animal group were subtracted from those dosed with MTX, as also performed for similar metabolites in urine, in order to focus the metabolite identification only on the features affected by MTX treatment.

In total, 24 compounds that were significantly altered by MTX treatment were putatively annotated (Table S3). Of these, 14 were dipeptides and one a tripeptide. Several of the other altered metabolites were acids, namely nicotinic acid, 3oxocholic acid, 4-ketoretinoic acid and $\mathrm{N}$-acetyl-neuraminic acid (Figure $2 \mathrm{~b}$ ). From the cluster analysis of the altered metabolites two main clusters of compounds were observed. One composed of four compounds which decreased through time in each of the MTX dose groups, and another group composed of the features which increased. Within the cluster of compounds increasing, some were observed to do so only in rats treated with $100 \mathrm{mg} / \mathrm{kg}$ of MTX (especially the dipeptides containing leucine or isoleucine, as well as glycyl-phenylalanine, prolyl-asparagine and prostaglandin B3), while others were significantly increased in animals receiving $40 \mathrm{mg} / \mathrm{kg}$. Thus, in the case of animals treated with $40 \mathrm{mg} / \mathrm{kg}$ of MTX, leucyl-glutamine, 4-ketoretinoic acid, $N$-acetyl-neuraminic acid, and glutamate increased over time even more than was the case of those dosed at $100 \mathrm{mg} / \mathrm{kg}$ (particularly leucylglutamine and 4-ketoretinoic acid). Similarly, serinyl-proline, nicotinic acid, histidinyl-proline, and 3-oxocholic acid decreased even more dramatically in the fecal extracts from animals treated with $40 \mathrm{mg} / \mathrm{kg}$.

\section{MTX Effects on the Fecal Microbiota}

The effect of MTX on the fecal microbiota Bray-Curtis PCoA analysis was used to assess $\beta$-diversity within the data set (Figure S8). No, or only small, differences were observed when comparing the PCoA plots of the predose and the 6-12 $\mathrm{h}$ postdose time points for all the dose-related groups. Greater variance was seen $36-48 \mathrm{~h}$ postdose compared to the predose time point across all groups; however, this was also observed in the control group. This dissimilarity was particularly marked for the fecal samples of the animals treated with $100 \mathrm{mg} / \mathrm{kg}$. $\alpha$ Diversity was also assessed using the Inverse Simpson index. The averaged $\alpha$ diversity is reported in Table S4. The $\alpha$ diversity of the untreated animals $(0 \mathrm{mg} / \mathrm{kg})$ was stable over time. However, for the animals dosed at $10 \mathrm{mg} / \mathrm{kg}$, the $\alpha$ diversity of their fecal samples had increased in the 6-12 h post MTX-dose samples before decreasing by $36-48 \mathrm{~h}$ post MTX-dose. In comparison, the $\alpha$ diversity of the fecal samples of the animals administered with $40 \mathrm{mg} / \mathrm{kg}$ MTX decreased at $6-12 \mathrm{~h}$ postdose and stabilized at $36-48 \mathrm{~h}$ postdose, which was not the case for the animals that received $100 \mathrm{mg} / \mathrm{kg}$ of the drug, where $\alpha$ diversity progressively decreased over the $48 \mathrm{~h}$ following MTX administration.

As both $\beta$ and $\alpha$ diversity displayed a stronger shift in the $36-48 \mathrm{~h}$ collection point, the taxonomic profiles of each of the MTX dose-related groups were aggregated and plotted for the pre- and $48 \mathrm{~h}$ postdose periods only (Figure 3 ). The fecal microbiota of the animal group dosed with $100 \mathrm{mg} / \mathrm{kg}$ of MTX provided the most notable microbial shift, albeit based on only a limited number of samples $(N=2)$ obtained due to diarrhea (see caption to Figure 3), with a striking increase in the relative abundance of Peptostreptococcaceae and Porphyromonadaceae and a decrease in the relative abundance of Ruminococcaceae. This alteration appears to have been dose dependent as it was not seen in the animals treated with 0 or $10 \mathrm{mg} / \mathrm{kg}$ of MTX but was visible in animals receiving $40 \mathrm{mg} / \mathrm{kg}$ (again, based on $N=3$ ) of the drug. A microbial shift was also observed over time for the vehicle-only treated group $(0 \mathrm{mg} / \mathrm{kg})$, with an increase in the relative abundance of unclassified Firmicutes and a decrease in the relative abundance of Erysipelotrichaceae. This change was also noted in animals that received 10 and 40 $\mathrm{mg} / \mathrm{kg}$ of MTX, but not those treated with $100 \mathrm{mg} / \mathrm{kg}$ of MTX, where the reverse occurred. Also, a decrease in the 

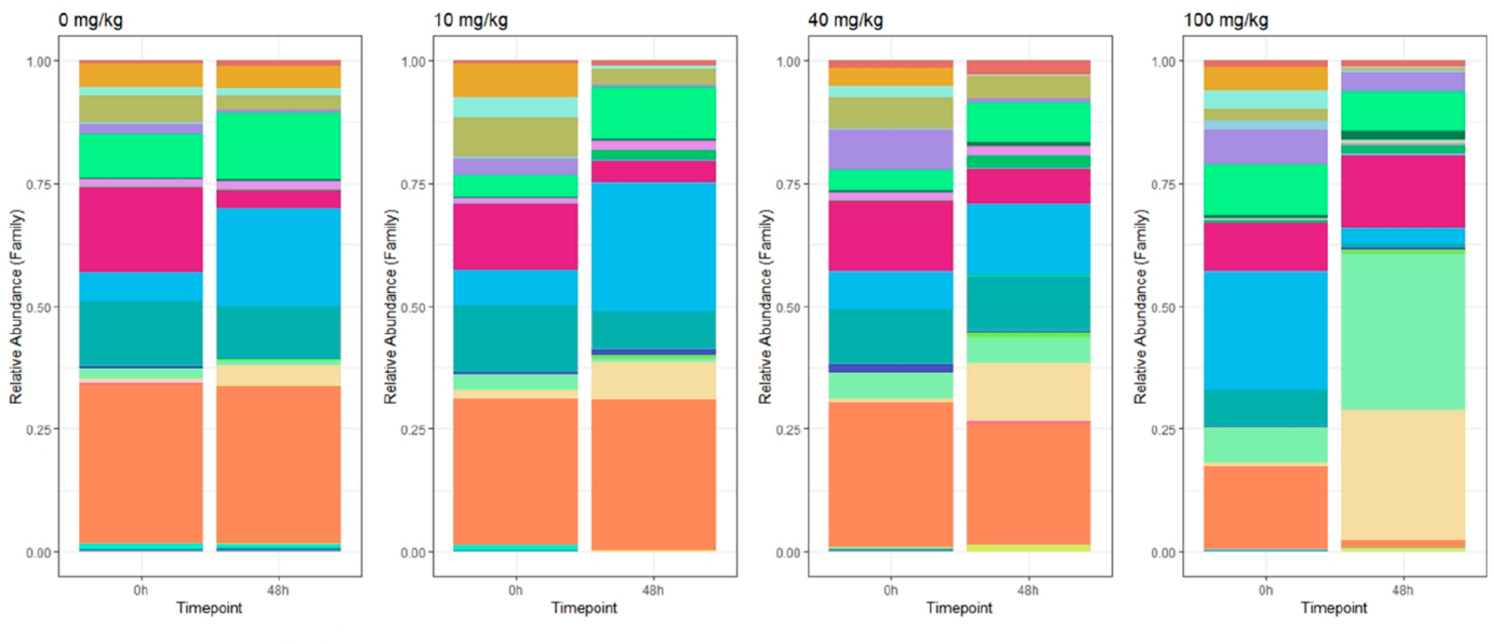

\begin{tabular}{llll} 
Family & & \\
Acidaminococcaceae & Coriobacteriaceae & Mollicutes_unclassified \\
\cline { 2 - 3 } Actinobacteria_unclassified & Corynebacteriaceae & Neisseriaceae \\
Aerococcaceae & Deferribacteraceae & Pasteurellaceae \\
\hline Alphaproteobacteria_unclassified & Deltaproteobacteria_unclassified & Peptostreptococcaceae \\
Anaeroplasmataceae & Desulfovibrionaceae & Porphyromonadaceae \\
Bacilli_unclassified & Enterobacteriaceae & Prevotellaceae \\
\hline Bacteria_unclassified & Enterococcaceae & Proteobacteria_unclassified \\
Bacteroidaceae & Erysipelotrichaceae & Rikenellaceae \\
Bacteroidales_unclassified & Eubacteriaceae & Ruminococcaceae \\
Bacteroidetes_unclassified & Firmicutes_unclassified & Staphylococcaceae \\
Bifidobacteriaceae & Flavobacteriaceae & Streptococcaceae \\
Carnobacteriaceae & Fusobacteriaceae & Sutterellaceae \\
\hline Clostridia_unclassified & Lachnospiraceae & Veillonellaceae \\
Clostridiaceae_1 & Lactobacillaceae & Verrucomicrobiaceae \\
Clostridiales_unclassified & Micrococcaceae & Victivallaceae
\end{tabular}

Figure 3. Family level taxonomic profiles (relative abundance) of the fecal microbiota of the rodents in each of the MTX dose-related groups preand post-MTX treatment $(0 \mathrm{~h}$ and 36-48 h, respectively). For the untreated animals, $N=4$ at $0 \mathrm{~h}$ and $N=3$ at $48 \mathrm{~h}$; for the animals which received a dose of $10 \mathrm{mg} / \mathrm{kg}, N=8$ at $0 \mathrm{~h}$ and $N=3$ at $48 \mathrm{~h}$; for the animals which received a dose of $40 \mathrm{mg} / \mathrm{kg}, N=8$ at $0 \mathrm{~h}$ and $N=4$ at $48 \mathrm{~h}$; for the animals which received a dose of $100 \mathrm{mg} / \mathrm{kg}, N=9$ at $0 \mathrm{~h}$ and $N=2$ at $48 \mathrm{~h}$.
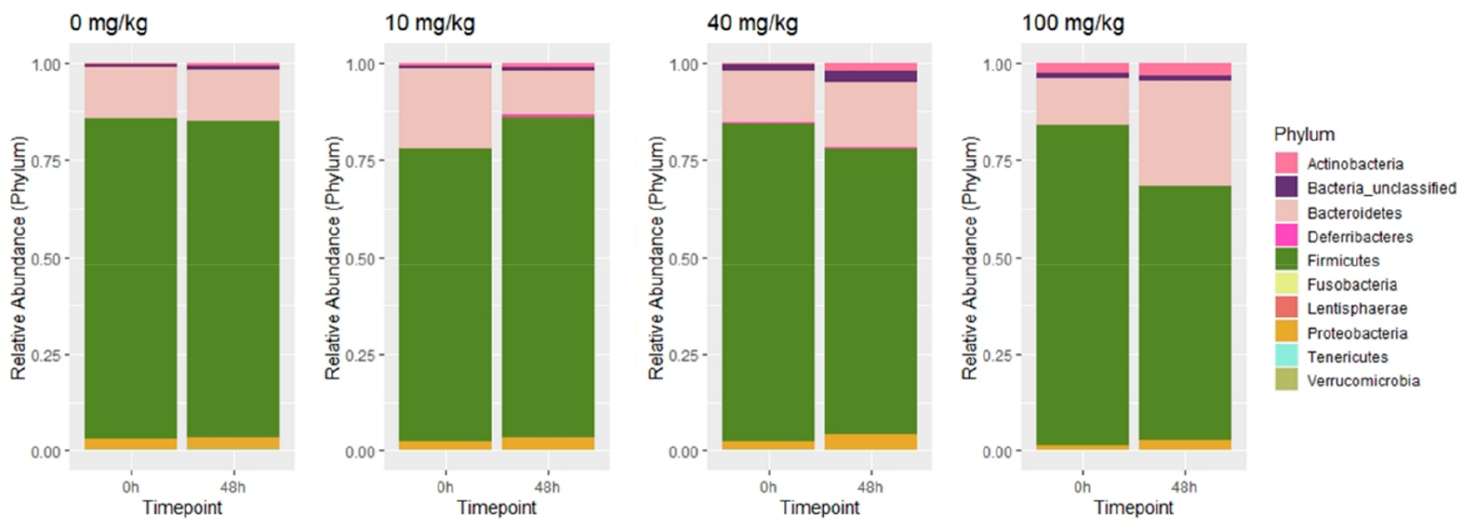

Figure 4. Taxonomic profiles of the fecal samples of the rodents representing the relative abundance of the phyla in each of the MTX dose-related group pre- and post-MTX treatment $(0$ and $48 \mathrm{~h}$, respectively). For the untreated animals, $N=4$ at $0 \mathrm{~h}$ and $N=3$ at $48 \mathrm{~h}$; for the animals which received a dose of $10 \mathrm{mg} / \mathrm{kg}, N=8$ at $0 \mathrm{~h}$ and $N=3$ at $48 \mathrm{~h}$; for the animals which received a dose of $40 \mathrm{mg} / \mathrm{kg}, N=8$ at $0 \mathrm{~h}$ and $N=4$ at $48 \mathrm{~h}$; for the animals which received a dose of $100 \mathrm{mg} / \mathrm{kg}, N=9$ at $0 \mathrm{~h}$ and $N=2$ at $48 \mathrm{~h}$.

relative abundance of Bacteroidaceae, unclassified Bacteroidales, Bacteroidetes, and Mollicutes was observed for the MTX-treated rats, particularly the $100 \mathrm{mg} / \mathrm{kg}$-dosed animals, compared to the control group $(0 \mathrm{mg} / \mathrm{kg})$.

Taxonomic profiles of the animals over time following MTX administration were also assessed at phylum level (Figure 4). A different trend was observed in response to the lowest and highest doses of MTX in changes at this level with, at the 10 $\mathrm{mg} / \mathrm{kg}$ dose, a decrease in the relative abundance of
Bacteroidetes and an increase in the relative abundance of Firmicutes was observed. Conversely, at doses of $40 \mathrm{mg} / \mathrm{kg}$ there was an increase in the relative abundance of Bacteroidetes and a decrease in the relative abundance of Firmicutes, which was exaggerated in the animals treated with $100 \mathrm{mg} / \mathrm{kg}$ of MTX. 


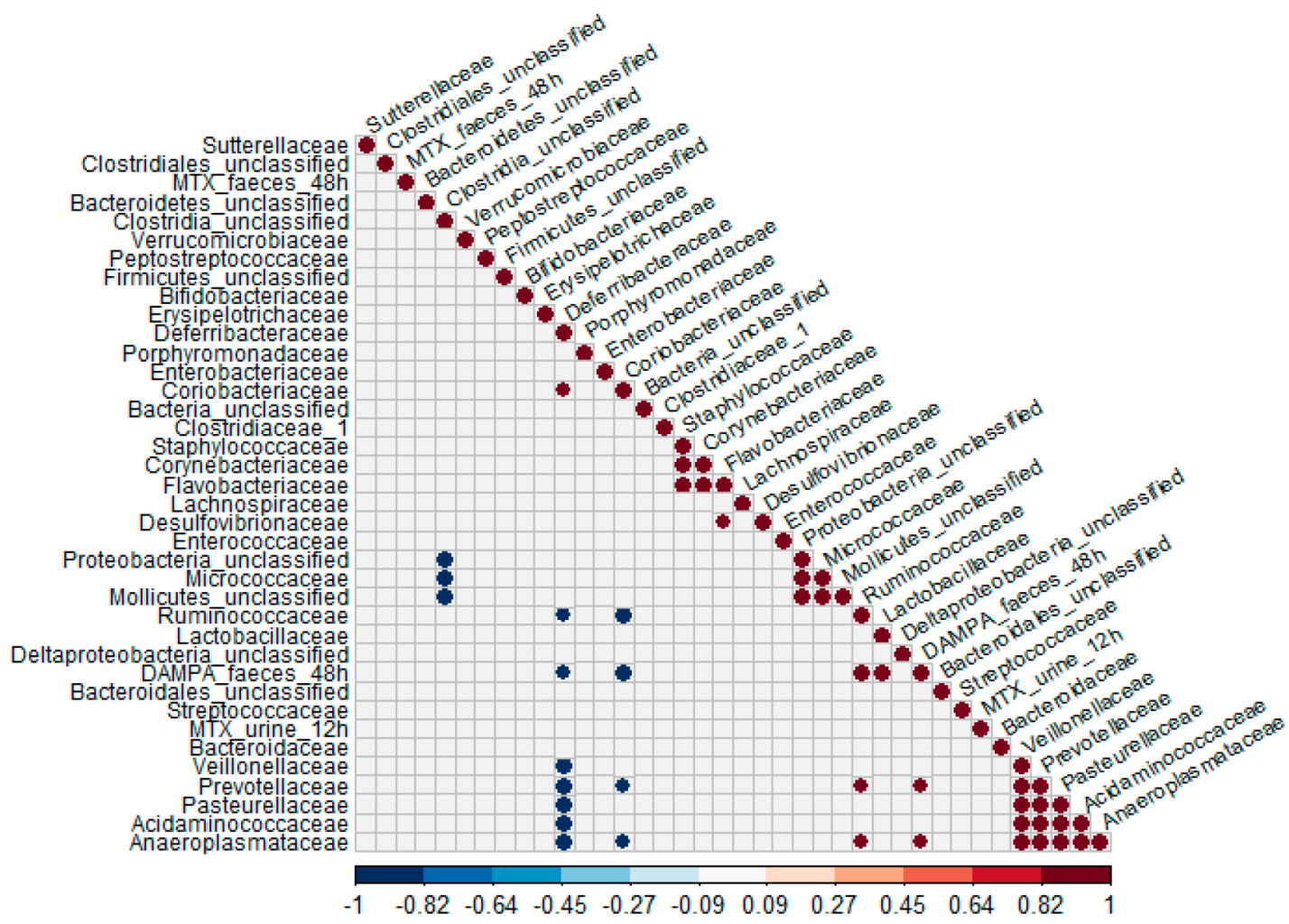

Figure 5. Correlogram integrating the bacteria present pre-MTX-dose in the $100 \mathrm{mg} / \mathrm{kg}$ dose animals fecal samples at family level with MTX concentration measured in urine at 6-12 h and in feces at 36-48 h, as well as the quantity of DAMPA excreted in feces samples at 36-48 h. This Figure shows only the significant correlations only $(p<0.05)$, highlighting positive correlations for DAMPA excreted in feces with Prevotellaceae, Anaeroplasmataceae, Ruminococcaceae, and Lactobacillaceae and a negative correlation with Deferribacteraceae and Coriobacteriaceae ( $N=3$ animals).

\section{Correlation Analysis}

Correlation analyses between the bacterial families present in the fecal samples of the $100 \mathrm{mg} / \mathrm{kg}$ dose animals prior to MTX treatment, with the quantity of MTX measured in urine at $12 \mathrm{~h}$ and in feces at $36-48 \mathrm{~h}$, as well as the quantity of DAMPA excreted in feces samples between 36 and $48 \mathrm{~h}$, were undertaken (Figure 5). The excretion of DAMPA in feces was positively correlated with the predose relative abundance of Prevotellaceae, Anaeroplasmataceae, Ruminococcaceae, and Lactobacillaceae, and negatively correlated with the predose relative abundance of Deferribacteraceae and Coriobacteriaceae. Other significant bacterial-metabolite linkages included a negative correlation between Deferribacteraceae and the bacterial families Veillonellaceae, Prevotellaceae, Pasteurellaceae, Acidaminococcaceae, and Anaeroplasmataceae (five families showing positive correlations with one another) and Ruminococcaceae (Figure 5). Similarly, the Coriobacteriaceae were negatively correlated with Prevotellaceae, Anaeroplasmataceae, and Ruminococcaceae (Figure 5).

Correlation analyses between the bacterial families present in the fecal samples of these high dose animals prior to MTX treatment, with the relative intensities of the putatively annotated metabolites measured in urine and in feces at 36$48 \mathrm{~h}$ were also performed (Figure 6). The relative abundances of Prevotellaceae and Anaeroplasmataceae, previously found to be positively correlated with the excretion of DAMPA in feces at $48 \mathrm{~h}$ (Figure 5), were found positively correlated with glutamate excreted in feces and 5-hydroxyindole acetic acid in urine at $48 \mathrm{~h}$ (Figure 6). Similarly, the relative abundances of Defferibacteraceae and Coriobacteriaceae, previously found to be negatively correlated with the excretion of DAMPA in feces at
$48 \mathrm{~h}$ (Figure 5), were found positively correlated with glutamate excreted in feces and 5-hydroxyindole acetic acid in urine at $48 \mathrm{~h}$ (Figure 6). The relative abundances of Prevotellaceae and Anaeroplasmataceae were negatively correlated with the relative abundances of Defferibacteraceae and Coriobacteriaceae in both analyses (Figures 5 and 6). Finally, the relative abundance of Lactobacillaceae, previously found to be positively correlated with the excretion of DAMPA in feces at $48 \mathrm{~h}$ (Figure 5), was also positively correlated with the amount of the putatively annotated $\mathrm{N}$-methyl-4-aminobenzoate excreted in urine at $48 \mathrm{~h}$. In addition, the relative abundance of Ruminococcaceae were also positively correlated with the peak putatively annotated as methionine sulfoximine excreted in urine at $48 \mathrm{~h}$ (Figure 6). However, as with all putative assignments, caution needs to be exercised with this structure which has, as noted above, previously only been positively identified in plants, ${ }^{49}$ or flour treated with $\mathrm{NCl}_{3} .{ }^{50}$

Correlation analyses between the quantity of MTX measured in urine at $12 \mathrm{~h}$ and in feces at $36-48 \mathrm{~h}$, as well as the quantity of DAMPA excreted in feces samples between 36 and $48 \mathrm{~h}$, with the relative intensities of the putatively annotated endogenous metabolites present in the urine and fecal samples of these high dose animals prior to MTX treatment, were also made (Figure S9). The excretion of MTX in feces at $48 \mathrm{~h}$ was found to negatively correlate with prolylasparagine in feces and 5-hydroxyindoleacetic acid in the urine samples of the predose animals. At $48 \mathrm{~h} 5$-hydroxyindoleacetic acid was found to be positively correlated with the relative abundances of Prevotellaceae and Anaeroplasmataceae pretreatment and negatively correlated with the relative abundances of Deferribacteraceae and Coriobacteriaceae pretreatment (Figure 


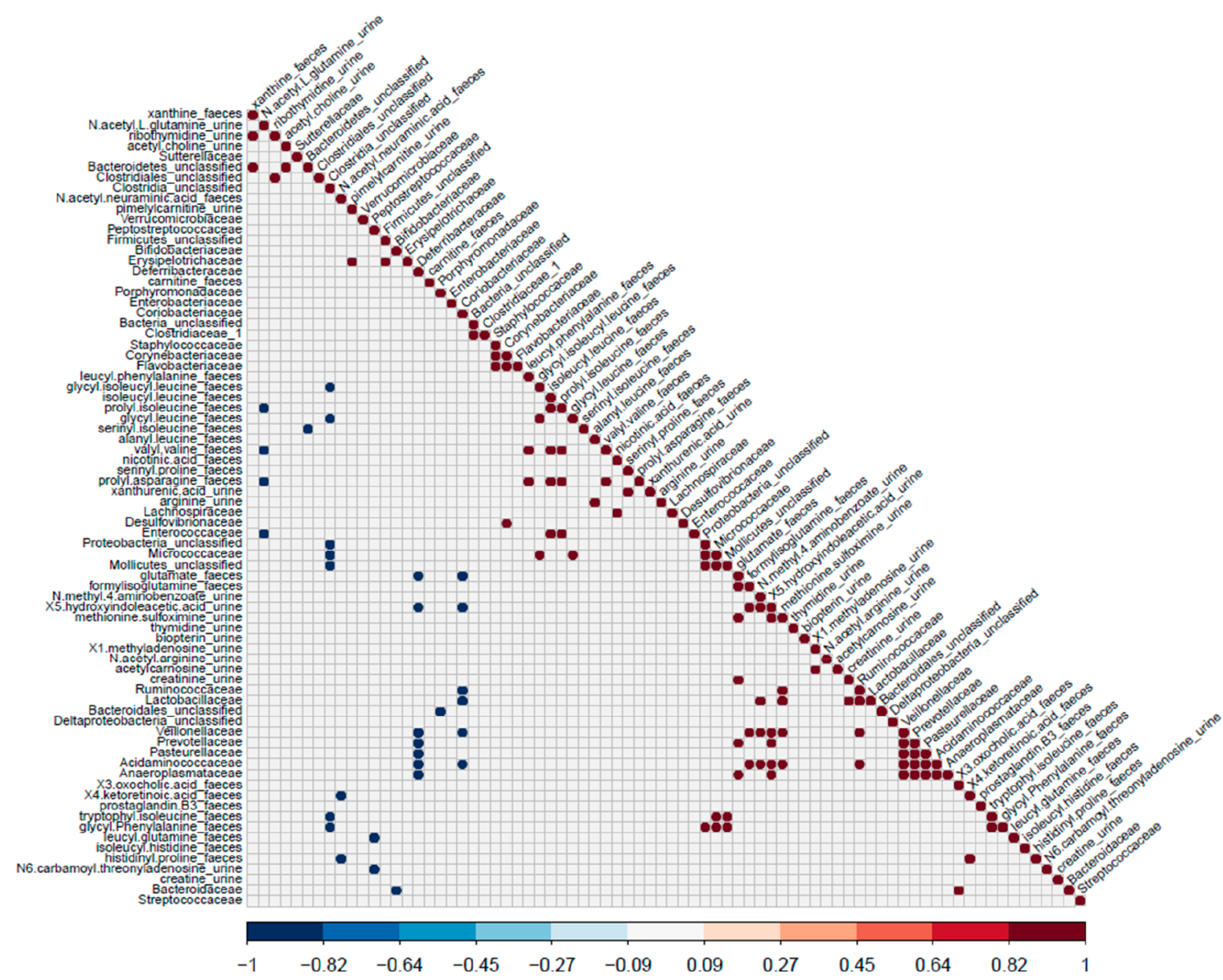

Figure 6. Correlogram integrating the bacteria present pre-MTX-dose in the fecal samples at family level with putatively annotated compounds excreted in urine and feces samples at 36-48 h. This Figure shows only significant correlations $(p<0.05)$ showing positive correlations between the relative abundances of Prevotellaceae, Anaeroplasmataceae, and glutamate in feces and 5-hydroxyindole acetic acid in urine at $48 \mathrm{~h}$, and negative correlation of the same metabolites with the relative abundance of Deferribacteraceae and Coriobacteriaceae. $N=3$ animals.

6), themselves showing the same correlative patterns with the excretion of DAMPA in feces at $48 \mathrm{~h}$ (Figure 5). The excretion of MTX in urine at $12 \mathrm{~h}$ was found to be positively correlated with the excretion of 4-ketoretinoic acid and nicotinic acid in the feces samples of the predose animals (Figure S9).

\section{DISCUSSION}

As indicated in the Introduction, in clinical use MTX has proved to be an effective treatment in diseases such as rheumatoid arthritis (RA), where it is a first line therapy, and in many cancers. ${ }^{1,2}$ But therapeutic responses can be variable and unpredictable and drug related (and dose limiting) toxicity ${ }^{3,29}$ (to liver, kidney and GI tract as well as myelosuppression) is also observed. The rat has proved to be a popular model species for studying the effects of MTX in vivo, including toxicity, ${ }^{51}$ and appears also to show some agreement with humans with respect to the pharmacokinetics of both MTX and 7-OH-MTX. ${ }^{51}$

A combination of targeted and untargeted UPLC-MS-based metabolic profiling approaches were used to assess the biotransformation of MTX and determine its wider biochemical impact on the urinary and fecal metabolomes of rats. In parallel, the fecal microbial profiles were defined to characterize the bidirectional interplay between MTX and the microbiota.

The untargeted UPLC-MS results highlighted a number of biochemical pathways that appeared to be affected by MTX, particularly at the $100 \mathrm{mg} / \mathrm{kg}$ dose. For example, MTX inhibits dihydrofolate reductase (DHFR), and therefore the production of the tetrahydrofolate, required for purine and pyrimidine synthesis, affects DNA synthesis (as well as the transmethylation of phospholipids and proteins ${ }^{52}$ ). MTX also inhibits thymidine synthesis when the free plasma concentration of MTX exceeds $10 \mathrm{nM}^{53}$ This is reflected in the observed reduction in the excretion of the pyrimidine nucleosides ribothymidine and thymidine after MTX administration. In contrast, the administration of $100 \mathrm{mg} / \mathrm{kg} \mathrm{MTX}$ increased the excretion of purine nucleoside-related compounds, such as N6-carbamoyl-threonyladenosine and 1methyl-adenosine, which is likely to be a consequence of the increased adenosine release previously reported as an antiinflammatory property of MTX. ${ }^{54}$ In addition, the amounts of xanthine, a purine base produced following the degradation of adenosine monophosphate to uric acid, increased in the feces following MTX exposure.

The urinary excretion of creatinine and its precursor creatine increased following MTX treatment (Figure 2). Creatine was previously found to be increased in the urine samples when the metabolic profile of urine from this study was studied by ${ }^{1} \mathrm{H}$ NMR spectroscopy. ${ }^{55}$ Interestingly, arginine, a precursor of creatine and a related metabolite $N$-acetylglutamine, also increased in the urine following MTX intake. Urinary N-acetylarginine was reduced, possibly due either to increased catabolism to produce arginine, or reduced consumption of 
arginine. Other urinary metabolites previously shown to be altered using ${ }^{1} \mathrm{H}$ NMR spectroscopic analysis following MTX treatment were methylamine, dimethylamine, TMAO, formiminoglutamic acid, alanine, phenylacetylglycine, succinate, citrate, 3-indoxyl sulfate, hippurate, and formate. ${ }^{55}$ MTXinduced elevations in the urinary excretion of creatine could also reflect disruptions to energy metabolism, as creatine is required for the production of phosphocreatine and thus ATP. The potential for MTX treatment to impact on energy metabolism has been previously been suggested ${ }^{55}$ and hypothesized to be related to reduced nutrient intake. This has been explained by MTX-induced gut epithelial loss and inflammation, morphological damage and malabsorption, previously reported in rats. ${ }^{29,56}$ Naruhashi et al. ${ }^{56}$ showed the disruption of intestinal peptide transporters, such as PEPT1, following MTX exposure, which may explain alterations in the fecal excretion of dipeptides following the 40 and $100 \mathrm{mg} / \mathrm{kg}$ MTX doses, reflecting an impairment in protein digestion or absorption. Increased concentrations of the metabolite 5-hydroxyindoleacetic acid, a metabolite of serotonin and gastroenteritis marker, ${ }^{57}$ in urine following MTX treatment could be explained by gastrointestinal toxicity. Similarly, in the fecal samples 4-ketoretinoic acid, involved in the maintenance of the epithelial tissue, ${ }^{58}$ and $\mathrm{N}$-acetylneuraminic acid, found in the gut mucus membrane (among others), were also found to be increased in amount in fecal extracts following MTX treatment. Other metabolites related to gut function and gut morphological toxicity were also perturbed by MTX intake. Fecal carnitine and urinary acetylcholine, which have both been shown to influence bile acid transport, ${ }^{59-62}$ were found to increase, while the bile acid 3-oxocholic acid was found to decrease in amount in fecal extracts, suggesting that targeted analysis for this class of metabolite might be warranted in future studies.

Fecal glutamate excretion increased over time following MTX treatment, which may have arisen by its release from the degradation of MTX during metabolism to DAMPA via the bacterial enzyme CPDG2. The semiquantification of MTX, 7OH-MTX and DAMPA in fecal water samples identified two distinct dose dependent effects. Thus, at the lowest MTX dose $(10 \mathrm{mg} / \mathrm{kg})$ both the drug and its metabolite 7-OH-MTX were excreted in the feces at $12 \mathrm{~h}$ while the microbial metabolite DAMPA was excreted between 12 to $48 \mathrm{~h}$. At the higher doses (40 or $100 \mathrm{mg} / \mathrm{kg}$ ) MTX and DAMPA excretion were delayed until $48 \mathrm{~h}$. The MTX excretion profile was found to be in agreement with a previous report of the same study using an alternative MS platform. ${ }^{29}$ Surprisingly, DAMPA excretion following the $100 \mathrm{mg} / \mathrm{kg}$ MTX dose was lower than that measured during the same time period for the animals administered with $40 \mathrm{mg} / \mathrm{kg}$ MTX. Saturation of CPDG2, the enzyme that cleaves glutamate from MTX to produce DAMPA, may explain this observation. Alternatively, a shift in the microbial community structure following the highest dose of MTX may reduce CPDG2 production or activity. Indeed, the fecal microbial profile was modulated by MTX with the notable changes having occurred by $48 \mathrm{~h}$ postdose being most pronounced at the $100 \mathrm{mg} / \mathrm{kg}$ dose. Interestingly, at the phylum level, changes observed between the pretreatment microbial profile and the microbial profile of the fecal samples collected at $48 \mathrm{~h}$ were different when the dose administrated was $10 \mathrm{mg} / \mathrm{kg}$ rather than $100 \mathrm{mg} / \mathrm{kg}$ of MTX. The microbial shift induced by $10 \mathrm{mg} / \mathrm{kg}$ of MTX included a decrease in Bacteroidetes and an increase in the Firmicutes. This was consistent with previous findings. ${ }^{24}$ Such changes in bacterial populations were then reversed at the $100 \mathrm{mg} / \mathrm{kg}$ dose level potentially explaining the recently reported dose related antimicrobial effect of MTX. ${ }^{24}$

Correlative studies show that DAMPA in feces was positively associated with Prevotellaceae, Anaeroplasmataceae, Ruminococcaceae, and Lactobacillaceae and was negatively associated with the relative abundance of Defferibacteraceae and Coriobacteriaceae (Figure 5). Interestingly, the relative abundances of Prevotellaceae and Anaeroplasmataceae as well as Defferibacteraceae and Coriobacteriaceae show the same correlative pattern with the excretion of glutamate in feces and the excretion of 5-hydroxyindole acetic acid in urine at 48 $\mathrm{h}$ (Figure 6). This could mean that the bacterial enzyme CPDG2, which cleaves MTX into DAMPA and glutamate, might be produced by bacteria from the families Prevotellaceae and Anaeroplasmataceae. However, 5-hydroxyindole acetic acid was found to be negatively correlated with the excretion of MTX in feces at $48 \mathrm{~h}$ (Figure S9). The excretion of the metabolite 4-ketoretinoic acid, involved in maintaining the gut epithelial layer, was found to be positively correlated with the excretion of MTX in urine at $12 \mathrm{~h}$ (Figure S9).

Prevotellaceae and Anaeroplasmataceae were strongly correlated with Ruminococcaceae and Lactobacillaceae, all of them positively correlating with DAMPA excretion in fecal samples. The relative abundances of these species also correlated with those of the Veillonellaceae, Pasteurellaceae, and Acidaminococcaceae. However, as shown in Figure 4, the relative abundance of the phylum Firmicutes decreased following a dose of 100 $\mathrm{mg} / \mathrm{kg}$ of MTX. This demonstrates that higher abundances of certain bacteria, mainly from the phylum Firmicutes, prior to administration of MTX were positively associated with higher concentrations of DAMPA in fecal samples. These bacteria could be producing the bacterial enzyme CPDG2, responsible for DAMPA production through MTX metabolism. Because the number of animals used in this study were kept to a minimum it is clear that further studies will be required to confirm these results. However, identifying the bacterial strains that can produce CPDG2 and determining the expression/ activity of this enzyme in the gut microbiome of patients prior to MTX administration could enable an individual's risk of MTX toxicity/reduced efficacy to be estimated prior to drug therapy. Clearly, in making this conjecture we recognize that it depends on limited data derived from a study conducted in a small number of normal rats rather than patients. Although the rat is thought to represent a suitable model for human with respect to MTX metabolism and toxicity ${ }^{51}$ it may be less suitable in terms of the gut microbiota, and the extrapolation of the present findings to clinical decision making clearly requires caution. However, if on investigation the results do translate to human disease, then patients identified as having low relative abundances of, e.g., Prevotellaceae and Anaeroplasmataceae could be identified before treatment was initiated. These patients could then either be proactively supplemented with CPDG2 to mitigate against MTX toxicity rather than it being used retroactively once toxicity develops as is current practice, ${ }^{2}$ or actively monitored so that treatment to limit MTX toxicity could be begun promptly.

\section{CONCLUSIONS}

When DAMPA, a nontoxic metabolite of MTX produced by the bacterial enzyme CPDG2, ${ }^{13,14}$ was quantified in fecal samples, its excretion positively correlated with the relative 
abundances of the Prevotellaceae and Anaeroplasmataceae. These bacterial families were themselves positively correlated with glutamate, which suggests that they are able to produce the CPDG2 enzyme. While further analysis is required to validate this hypothesis, determining the abundance of these bacteria in patients to inform subsequent chemotherapeutic treatment may contribute to the personalization of this strategy. Conversely, high doses of MTX perturbed the gut microbial community, increasing the relative abundance of Bacteroidetes and decreasing that of the Firmicutes. Perturbations in endogenous metabolites involved in gut disorder and gut epithelium or mucus maintenance such as 5hydroxyindoleacetic acid, 4-ketoretinoic acid, and $\mathrm{N}$-neuraminic acid were assumed to reflect toxicity. Chronic exposure to MTX could modify the composition and functional capacity of the microbiome with a subsequent impact on its ability to produce CPDG2 and detoxify the drug. A clearer understanding of this relationship in humans may have the potential to enhance the effectiveness of MTX therapy and minimize toxic outcomes.

\section{ASSOCIATED CONTENT}

\section{(s) Supporting Information}

The Supporting Information is available free of charge at https://pubs.acs.org/doi/10.1021/acs.jproteome.0c00230.

R Script for feature removal; Figure S1: Screenshot of TargetLynx V4.1 showing the three calibration curves for MTX, DAMPA, and 7-OH-MTX used during the fecal sample analysis; Figure S2: PCA score plots of the urine metabolic profile, plotted according to the dose of MTX administrated; Figure S3: CA-PLS-R (covariateadjusted projection to latent structures regression) analysis performed on the urine samples over the time points $0,12,24$, and $48 \mathrm{~h}$ for each of the MTX dosegroups; Figure S4: Skyline significance of the CA-PLSRegression models, performed on the urine samples for each of the MTX dose groups (0,10, 40, and $100 \mathrm{mg}$ / $\mathrm{kg}$ ), representing the number of features having a $q$-value less than 0.05 and which significantly increased (red color) or decreased (blue color) over time $(0,12,24$, and $48 \mathrm{~h})$; Figure S5: PCA score plots of the fecal metabolic profiles plotted according to the dose of MTX administered; Figure S6: CA-PLS-R (covariate-adjusted projection to latent structures regression) analysis performed over time $(0,12$, and $48 \mathrm{~h})$ on the fecal samples of each of the MTX dose groups; Figure S7: Skyline significance of the CA-PLS-Regression models, performed on fecal samples for each of the MTX dose groups $(0,10,40$, and $100 \mathrm{mg} / \mathrm{kg})$, representing the number of features having a $q$-value lower than 0.05 and which significantly increased (red color) or decreased (blue color) over time (0, 12 and $48 \mathrm{~h})$; Figure S8: $\beta$ diversity represented using PCoA of Bray-Curtis distance, displaying the similarity between the samples; Figure S9: Correlogram integrating the putatively annotated endogenous compounds excreted in urine and feces samples of the animals pre-MTX treatment with MTX concentration measured in urine at 6-12 h and in feces at $36-48 \mathrm{~h}$, as well as the quantity of DAMPA excreted in feces samples at $36-48 \mathrm{~h}$; Table S1: Number of reads per samples after 16S rRNA gene sequencing preprocessing and prior importation into phyloseq for data investigation; Table S2: Putative annotations for metabolites detected in the urine samples; Table S3: Putative annotations for metabolites detected in the fecal samples; Table S4: $\alpha$ Diversity values representing the microbial richness of each of the fecal samples of the animals according the MTX dose groups over time (PDF)

\section{AUTHOR INFORMATION}

\section{Corresponding Authors}

Marine P. M. Letertre - Department of Metabolism, Digestion and Reproduction, Faculty of Medicine, Imperial College, London SW7 2AZ, U.K.; (1) orcid.org/0000-0002-7777-1001; Email: m.letertre16@imperial.ac.uk

Ian D. Wilson - Department of Metabolism, Digestion and Reproduction, Faculty of Medicine, Imperial College, London SW7 2AZ, U.K.; 10 orcid.org/0000-0002-8558-7394; Email: i.wilson@imperial.ac.uk

Authors

Nyasha Munjoma - Waters Corporation, Wilmslow SK9 4AX, U.K.

Kate Wolfer - Department of Metabolism, Digestion and Reproduction, Faculty of Medicine, Imperial College, London SW7 2AZ, U.K.

Alexandros Pechlivanis - Department of Metabolism, Digestion and Reproduction, Faculty of Medicine, Imperial College, London SW7 2AZ, U.K.; Center for Interdisciplinary Research of the Aristotle University of Thessaloniki (KEDEK), 57001 Thessaloniki, Greece; Department of Chemistry, Aristotle University of Thessaloniki, 54124 Thessaloniki, Greece

Julie A. K. McDonald - Department of Metabolism, Digestion and Reproduction, Faculty of Medicine, Imperial College, London SW7 2AZ, U.K.

Rhiannon N. Hardwick - Department of Pharmacology and Toxicology, College of Pharmacy, University of Arizona, Tuscon, Arizona 85721, United States

Nathan J. Cherrington - Department of Pharmacology and Toxicology, College of Pharmacy, University of Arizona, Tuscon, Arizona 85721, United States

Muireann Coen - Department of Metabolism, Digestion and Reproduction, Faculty of Medicine, Imperial College, London SW7 2AZ, U.K.; Oncology Safety, Clinical Pharmacology \& Safety Sciences, R\&D, Astra Zeneca, Cambridge CB4 OWG, U.K.

Jeremy K. Nicholson - Australian National Phenome Centre, Health Futures Institute, Murdoch University, Murdoch, WA 6150, Australia

Lesley Hoyles - Department of Metabolism, Digestion and Reproduction, Faculty of Medicine, Imperial College, London SW7 2AZ, U.K.; Department of Biosciences, Nottingham Trent University, Nottingham NG11 8NS, U.K.; (1) orcid.org/00000002-6418-342X

Jonathan R. Swann - Department of Metabolism, Digestion and Reproduction, Faculty of Medicine, Imperial College, London SW7 2AZ, U.K.

Complete contact information is available at:

https://pubs.acs.org/10.1021/acs.jproteome.0c00230

\section{Author Contributions}

The manuscript was written through contributions from all authors. Animal experiment was performed by RNH. UPLC- 
MS experiments were performed by MPML and NM. Amplicon sequencing was performed by MPML and JAKM. UPLC-MS processing and interpretation was done by MPML, $\mathrm{KW}$, and AP. Amplicon sequencing interpretation was made by MPML and LH. Supervision was by JRS and IDW. All authors have given approval to the final version of the manuscript.

Notes

The authors declare no competing financial interest.

\section{ACKNOWLEDGMENTS}

This work was supported by the Servier Technologies through the STRATiGRAD Program of Imperial College London. The NPC database construction and matching were supported by the Medical Research Council and National Institute for Health Research [PI JKN, grant number MC_PC_12025] as the National Phenome Centre. LH was supported by the Medical Research Council [grant number MR/L01632X/1]. $\mathrm{RNH}$ and NJC were supported by the United States National Institutes of Health grants AI083927, ES007091, and ES006694. Infrastructure support for this work was provided by the NIHR Imperial Biomedical Research Centre.

\section{REFERENCES}

(1) Nathan, P. C.; Whitcomb, T.; Wolters, P. L.; Steinberg, S. M.; Balis, F. M.; Brouwers, P.; Hunsberger, S.; Feusner, J.; Sather, H.; Miser, J.; Odom, L. F.; Poplack, D.; Reaman, G.; Bleyer, W. A. Very High-Dose Methotrexate $(33.6 \mathrm{~g} / \mathrm{m}(2))$ as Central Nervous System Preventive Therapy for Childhood Acute Lymphoblastic Leukemia: Results of National Cancer Institute/Children's Cancer Group Trials CCG-191P, CCG-134P and CCG-144P. Leuk. Lymphoma 2006, 47 (12), 2488-2504.

(2) Rattu, M. A.; Shah, N.; Lee, J. M.; Pham, A. Q.; Marzella, N. Glucarpidase (Voraxaze), a Carboxypeptidase Enzyme for Methotrexate Toxicity. Pharm. Ther. 2013, 38 (12), 732-744.

(3) Buchen, S.; Ngampolo, D.; Melton, R. G.; Hasan, C.; Zoubek, A.; Henze, G.; Bode, U.; Fleischhack, G. Carboxypeptidase G2 Rescue in Patients with Methotrexate Intoxication and Renal Failure. Br. J. Cancer 2005, 92 (3), 480-487.

(4) Genestier, L.; Paillot, R.; Quemeneur, L.; Izeradjene, K.; Revillard, J.-P. Mechanisms of Action of Methotrexate. Immunopharmacology 2000, 47 (2-3), 247-257.

(5) Goldman, I. D.; Matherly, L. H. The Cellular Pharmacology of Methotrexate. Pharmacol. Ther. 1985, 28 (1), 77-102.

(6) Allegra, C. J.; Chabner, B. A.; Drake, J. C.; Lutz, R.; Rodbard, D.; Jolivet, J. Enhanced Inhibition of Thymidylate Synthase by Methotrexate Polyglutamates. J. Biol. Chem. 1985, 260 (17), 97209726.

(7) Allegra, C. J.; Hoang, K.; Yeh, G. C.; Drake, J. C.; Baram, J. Evidence for Direct Inhibition of de Novo Purine Synthesis in Human MCF-7 Breast Cells as a Principal Mode of Metabolic Inhibition by Methotrexate. J. Biol. Chem. 1987, 262 (28), 13520-13526.

(8) Nicholson, J. K.; Holmes, E.; Wilson, I. D. Gut Microorganisms, Mammalian Metabolism and Personalized Health Care. Nat. Rev. Microbiol. 2005, 3 (5), 431-438.

(9) Carmody, R. N.; Turnbaugh, P. J. Host-Microbial Interactions in the Metabolism of Therapeutic and Diet-Derived Xenobiotics. J. Clin. Invest. 2014, 124 (10), 4173-4181.

(10) Wilson, I. D.; Nicholson, J. K. Gut Microbiome Interactions with Drug Metabolism, Efficacy, and Toxicity. Transl Res. 2017, 179, 204.

(11) Alexander, J. L.; Wilson, I. D.; Teare, J.; Marchesi, J. R.; Nicholson, J. K.; Kinross, J. M. Gut Microbiota Modulation of Chemotherapy Efficacy and Toxicity. Nat. Rev. Gastroenterol. Hepatol. 2017, 14 (6), 356-365.
(12) Clarke, G.; Sandhu, K. V.; Griffin, B. T.; Dinan, T. G.; Cryan, J. F.; Hyland, N. P. Gut Reactions: Breaking Down XenobioticMicrobiome Interactions. Pharmacol. Rev. 2019, 71 (2), 198-224.

(13) Zaharko, D. S.; Bruckner, H.; Oliverio, V. T. Antibiotics Alter Methotrexate Metabolism and Excretion. Science 1969, 166 (3907), 887-888.

(14) Valerino, D. M.; Johns, D. G.; Zaharko, D. S.; Oliverio, V. T. Studies of the Metabolism of Methotrexate by Intestinal Flora. I. Identification and Study of Biological Properties of the Metabolite 4Amino-4-Deoxy-N 10 -Methylpteroic Acid. Biochem. Pharmacol. 1972, 21 (6), 821-831.

(15) Schmiegelow, K. Advances in Individual Prediction of Methotrexate Toxicity: A Review. Br. J. Haematol. 2009, 146 (5), 489-503.

(16) Widemann, B. C.; Sung, E.; Anderson, L.; Salzer, W. L.; Balis, F. M.; Monitjo, K. S.; McCully, C.; Hawkins, M.; Adamson, P. C. Pharmacokinetics and Metabolism of the Methotrexate Metabolite 2,4-Diamino-N 10-Methylpteroic Acid. J. Pharmacol. Exp. Ther. 2000, 294 (3), 894-901.

(17) Larimer, C. M.; Slavnic, D.; Pitstick, L. D.; Green, J. M. Comparison of Substrate Specificity of Escherichia Coli P-Aminobenzoyl-Glutamate Hydrolase with Pseudomonas Carboxypeptidase G. Adv. Enzyme Res. 2014, 2 (1), 39-48.

(18) Donehower, R. C.; Hande, K. R.; Drake, J. C.; Chabner, B. A. Presence of 2,4-Diamino-N10-Methylpteroic Acid after High-Dose Methotrexate. Clin. Pharmacol. Ther. 1979, 26 (1), 63-72.

(19) Widemann, B. C.; Balis, F. M.; Adamson, P. C. Dihydrofolate Reductase Enzyme Inhibition Assay for Plasma Methotrexate Determination Using a 96-Well Microplate Reader. Clin. Chem. 1999, 45 (2), 223-228.

(20) Widemann, B. C.; Jayaprakash, N.; Howard, S. C.; Daugherty, C.; Chauhan, N.; King, T.; Rush, J. Clinical Trial and Compassionate Use Experience with Glucarpidase for Methotrexate Toxicity. J. Clin. Oncol. 2012, 30 (15 suppl), 6530-6530.

(21) Svahn, T.; Mellgren, K.; Harila-Saari, A.; Åsberg, A.; Kanerva, J.; Jónsson, O.; Vaitkeviciene, G.; Stamm Mikkelssen, T.; Schmiegelow, K.; Heldrup, J. Delayed Elimination of High-Dose Methotrexate and Use of Carboxypeptidase G2 in Pediatric Patients during Treatment for Acute Lymphoblastic Leukemia. Pediatr. Blood Cancer 2017, 64 (7), No. e26395.

(22) Shea, B.; Swinden, M. V.; Tanjong Ghogomu, E.; Ortiz, Z.; Katchamart, W.; Rader, T.; Bombardier, C.; Wells, G. A.; Tugwell, P. Folic Acid and Folinic Acid for Reducing Side Effects in Patients Receiving Methotrexate for Rheumatoid Arthritis. Cochrane Database Syst. Rev. 2013, No. 5, CD000951.

(23) Maier, L.; Pruteanu, M.; Kuhn, M.; Zeller, G.; Telzerow, A.; Anderson, E. E.; Brochado, A. R.; Fernandez, K. C.; Dose, H.; Mori, H.; Patil, K. R.; Bork, P.; Typas, A. Extensive Impact of NonAntibiotic Drugs on Human Gut Bacteria. Nature 2018, 555 (7698), 623-628.

(24) Nayak, R. R.; Alexander, M.; Stapleton-Grey, K.; Ubeda, C.; Scher, J. U.; Turnbaugh, P. J. Perturbation of the Human Gut Microbiome by a Non-Antibiotic Drug Contributes to the Resolution of Autoimmune Disease. bioRxiv, April 5, 2019, DOI: 10.1101/ 600155 (accessed July 22, 2019).

(25) Zhang, X.; Zhang, D.; Jia, H.; Feng, Q.; Wang, D.; Liang, D.; Wu, X.; Li, J.; Tang, L.; Li, Y.; Lan, Z.; Chen, B.; Li, Y.; Zhong, H.; Xie, H.; Jie, Z.; Chen, W.; Tang, S.; Xu, X.; Wang, X.; Cai, X.; Liu, S.; Xia, Y.; Li, J.; Qiao, X.; Al-Aama, J. Y.; Chen, H.; Wang, L.; Wu, Q.; Zhang, F.; Zheng, W.; Li, Y.; Zhang, M.; Luo, G.; Xue, W.; Xiao, L.; Li, J.; Chen, W.; Xu, X.; Yin, Y.; Yang, H.; Wang, J.; Kristiansen, K.; Liu, L.; Li, T.; Huang, Q.; Li, Y.; Wang, J. The Oral and Gut Microbiomes Are Perturbed in Rheumatoid Arthritis and Partly Normalized after Treatment. Nat. Med. 2015, 21 (8), 895-905.

(26) Chen, J.; Wright, K.; Davis, J. M.; Jeraldo, P.; Marietta, E. V.; Murray, J.; Nelson, H.; Matteson, E. L.; Taneja, V. An Expansion of Rare Lineage Intestinal Microbes Characterizes Rheumatoid Arthritis. Genome Med. 2016, DOI: 10.1186/s13073-016-0299-7. 
(27) Bolin, J. T.; Filman, D. J.; Matthews, D. A.; Hamlin, R. C.; Kraut, J. Crystal Structures of Escherichia coli and Lactobacillus casei Dihydrofolate Reductase Refined at 1.7 A Resolution. I. General Features and Binding of Methotrexate. J. Biol. Chem. 1982, 257 (22), 13650-13662.

(28) Fijlstra, M.; Ferdous, M.; Koning, A. M.; Rings, E. H. H. M.; Harmsen, H. J. M.; Tissing, W. J. E. Substantial Decreases in the Number and Diversity of Microbiota during Chemotherapy-Induced Gastrointestinal Mucositis in a Rat Model. Support Care Cancer 2015, 23 (6), 1513-1522.

(29) Hardwick, R. N.; Clarke, J. D.; Lake, A. D.; Canet, M. J.; Anumol, T.; Street, S. M.; Merrell, M. D.; Goedken, M. J.; Snyder, S. A.; Cherrington, N. J. Increased Susceptibility to MethotrexateInduced Toxicity in Nonalcoholic Steatohepatitis. Toxicol. Sci. 2014, 142 (1), 45-55.

(30) Gika, H. G.; Theodoridis, G. A.; Wingate, J. E.; Wilson, I. D. Within-Day Reproducibility of an HPLC-MS-Based Method for Metabonomic Analysis: Application to Human Urine. J. Proteome Res. 2007, 6 (8), 3291-3303.

(31) Broadhurst, D.; Goodacre, R.; Reinke, S. N.; Kuligowski, J.; Wilson, I. D.; Lewis, M. R.; Dunn, W. B. Guidelines and Considerations for the Use of System Suitability and Quality Control Samples in Mass Spectrometry Assays Applied in Untargeted Clinical Metabolomic Studies. Metabolomics 2018, 14 (6), 72.

(32) Gray, N.; Adesina-Georgiadis, K.; Chekmeneva, E.; Plumb, R. S.; Wilson, I. D.; Nicholson, J. K. Development of a Rapid Microbore Metabolic Profiling Ultraperformance Liquid Chromatography-Mass Spectrometry Approach for High-Throughput Phenotyping Studies. Anal. Chem. 2016, 88 (11), 5742-5751.

(33) Lewis, M. R.; Pearce, J. T. M.; Spagou, K.; Green, M.; Dona, A. C.; Yuen, A. H. Y.; David, M.; Berry, D. J.; Chappell, K.; HornefferVan der Sluis, V.; Shaw, R.; Lovestone, S.; Elliott, P.; Shockcor, J.; Lindon, J. C.; Cloarec, O.; Takats, Z.; Holmes, E.; Nicholson, J. K. Development and Application of Ultra-Performance Liquid Chromatography-TOF MS for Precision Large Scale Urinary Metabolic Phenotyping. Anal. Chem. 2016, 88 (18), 9004-9013.

(34) Mullish, B. H.; Pechlivanis, A.; Barker, G. F.; Thursz, M. R.; Marchesi, J. R.; McDonald, J. A. K. Functional Microbiomics: Evaluation of Gut Microbiota-Bile Acid Metabolism Interactions in Health and Disease. Methods 2018, 149, 49-58.

(35) Smith, C. A.; Want, E. J.; O’Maille, G.; Abagyan, R.; Siuzdak, G. XCMS: Processing Mass Spectrometry Data for Metabolite Profiling Using Nonlinear Peak Alignment, Matching, and Identification. Anal. Chem. 2006, 78 (3), 779-787.

(36) Tautenhahn, R.; Böttcher, C.; Neumann, S. Highly Sensitive Feature Detection for High Resolution LC/MS. BMC Bioinf. 2008, 9 (1), 504.

(37) Benton, H. P.; Want, E. J.; Ebbels, T. M. D. Correction of Mass Calibration Gaps in Liquid Chromatography-Mass Spectrometry Metabolomics Data. Bioinformatics 2010, 26 (19), 2488-2489.

(38) Kessner, D.; Chambers, M.; Burke, R.; Agus, D.; Mallick, P. ProteoWizard: Open Source Software for Rapid Proteomics Tools Development. Bioinformatics 2008, 24 (21), 2534-2536.

(39) Veselkov, K. A.; Vingara, L. K.; Masson, P.; Robinette, S. L.; Want, E.; Li, J. V.; Barton, R. H.; Boursier-Neyret, C.; Walther, B.; Ebbels, T. M.; Pelczer, I.; Holmes, E.; Lindon, J. C.; Nicholson, J. K. Optimized Preprocessing of Ultra-Performance Liquid Chromatography/Mass Spectrometry Urinary Metabolic Profiles for Improved Information Recovery. Anal. Chem. 2011, 83 (15), 5864-5872.

(40) Rocke, D. M.; Durbin, B. Approximate Variance-Stabilizing Transformations for Gene-Expression Microarray Data. Bioinformatics 2003, 19 (8), 966-972.

(41) Posma, J. M.; Garcia-Perez, I.; Ebbels, T. M. D.; Lindon, J. C.; Stamler, J.; Elliott, P.; Holmes, E.; Nicholson, J. K. Optimized Phenotypic Biomarker Discovery and Confounder Elimination via Covariate-Adjusted Projection to Latent Structures from Metabolic Spectroscopy Data. J. Proteome Res. 2018, 17 (4), 1586-1595.

(42) Benjamini, Y.; Hochberg, Y. Controlling the False Discovery Rate: A Practical and Powerful Approach to Multiple Testing. Journal of the Royal Statistical Society: Series B (Methodological) 1995, 57 (1), 289-300.

(43) Gil de la Fuente, A.; Godzien, J.; Fernández López, M.; Rupérez, F. J.; Barbas, C.; Otero, A. Knowledge-Based Metabolite Annotation Tool: CEU Mass Mediator. J. Pharm. Biomed. Anal. 2018, $154,138-149$.

(44) Sumner, L. W.; Amberg, A.; Barrett, D.; Beale, M. H.; Beger, R.; Daykin, C. A.; Fan, T. W.-M.; Fiehn, O.; Goodacre, R.; Griffin, J. L.; Hankemeier, T.; Hardy, N.; Harnly, J.; Higashi, R.; Kopka, J.; Lane, A. N.; Lindon, J. C.; Marriott, P.; Nicholls, A. W.; Reily, M. D.; Thaden, J. J.; Viant, M. R. Proposed Minimum Reporting Standards for Chemical Analysis Chemical Analysis Working Group (CAWG) Metabolomics Standards Initiative (MSI). Metabolomics 2007, 3 (3), 211-221.

(45) Liu, C. M.; Aziz, M.; Kachur, S.; Hsueh, P.-R.; Huang, Y.-T.; Keim, P.; Price, L. B. BactQuant: An Enhanced Broad-Coverage Bacterial Quantitative Real-Time PCR Assay. BMC Microbiol. 2012, 12, 56.

(46) Kozich, J. J.; Westcott, S. L.; Baxter, N. T.; Highlander, S. K.; Schloss, P. D. Development of a Dual-Index Sequencing Strategy and Curation Pipeline for Analyzing Amplicon Sequence Data on the MiSeq Illumina Sequencing Platform. Appl. Environ. Microbiol. 2013, 79 (17), 5112-5120.

(47) Wang, Q.; Garrity, G. M.; Tiedje, J. M.; Cole, J. R. Nä̈ve Bayesian Classifier for Rapid Assignment of RRNA Sequences into the New Bacterial Taxonomy. Appl. Environ. Microbiol. 2007, 73 (16), $5261-5267$.

(48) McMurdie, P. J.; Holmes, S. Waste Not, Want Not: Why Rarefying Microbiome Data Is Inadmissible. PLoS Comput. Biol. 2014, 10 (4), No. e1003531.

(49) Jeannoda, V. L.; Rakoto-Ranoromalala, D. A.; Valisolalao, J.; Creppy, E. E.; Dirheimer, G. Natural Occurrence of Methionine Sulfoximine in the Connaraceae Family. J. Ethnopharmacol. 1985, 14 (1), 11-17.

(50) Bentley, H. R.; McDermott, E. E.; Whitehead, J. K. Action of Nitrogen Trichloride on Proteins: A Synthesis of the Toxic Factor from Methionine. Nature 1950, 165 (4201), 735-735.

(51) Bremnes, R. M.; Slørdal, L.; Wist, E.; Aarbakke, J. DoseDependent Pharmacokinetics of Methotrexate and 7-Hydroxymethotrexate in the Rat in Vivo. Cancer Res. 1989, 49 (22), 6359-6364.

(52) Beh, S. C.; Kildebeck, E.; Narayan, R.; Desena, A.; Schell, D.; Rowe, E. S.; Rowe, V.; Burns, D.; Whitworth, L.; Frohman, T. C.; Greenberg, B.; Frohman, E. M. High-Dose Methotrexate with Leucovorin Rescue: For Monumentally Severe CNS Inflammatory Syndromes. J. Neurol. Sci. 2017, 372, 187-195.

(53) Chabner, B. A.; Young, R. C. Threshold Methotrexate Concentration for In Vivo Inhibition of DNA Synthesis in Normal and Tumorous Target Tissues. J. Clin. Invest. 1973, 52 (8), 18041811.

(54) Cronstein, B. N.; Naime, D.; Ostad, E. The Antiinflammatory Mechanism of Methotrexate. Increased Adenosine Release at Inflamed Sites Diminishes Leukocyte Accumulation in an in Vivo Model of Inflammation. J. Clin. Invest. 1993, 92 (6), 2675-2682.

(55) Kyriakides, M.; Hardwick, R. N.; Jin, Z.; Goedken, M. J.; Holmes, E.; Cherrington, N. J.; Coen, M. Systems Level Metabolic Phenotype of Methotrexate Administration in the Context of NonAlcoholic Steatohepatitis in the Rat. Toxicol. Sci. 2014, 142 (1), 105116.

(56) Naruhashi, K.; Nadai, M.; Nakao, M.; Suzuki, N.; Nabeshima, T.; Hasegawa, T. Changes In Absorptive Function Of Rat Intestine Injured By Methotrexate. Clin. Exp. Pharmacol. Physiol. 2000, 27 (12), 980-986.

(57) Ilkhanizadeh, B.; Owji, A. A.; Tavangar, S. M.; Vasei, M.; Tabei, S. M. Spot Urine 5-Hydroxy Indole Acetic Acid and Acute Appendicitis. Hepatogastroenterology 2001, 48 (39), 609-613.

(58) Heise, R.; Mey, J.; Neis, M. M.; Marquardt, Y.; Joussen, S.; Ott, H.; Wiederholt, T.; Kurschat, P.; Megahed, M.; Bickers, D. R.; Merk, H. F.; Baron, J. M. Skin Retinoid Concentrations Are Modulated by CYP26AI Expression Restricted to Basal Keratinocytes in Normal 
Human Skin and Differentiated 3D Skin Models. J. Invest. Dermatol. 2006, 126 (11), 2473-2480.

(59) Krähenbühl, S.; Reichen, J. Carnitine Metabolism in Patients with Chronic Liver Disease. Hepatology 1997, 25 (1), 148-153.

(60) Kirsch, S. H.; Herrmann, W.; Rabagny, Y.; Obeid, R. Quantification of Acetylcholine, Choline, Betaine, and Dimethylglycine in Human Plasma and Urine Using Stable-Isotope Dilution Ultra Performance Liquid Chromatography-Tandem Mass Spectrometry. J. Chromatogr. B: Anal. Technol. Biomed. Life Sci. 2010, 878 (32), 33383344.

(61) Lips, K. S.; Wunsch, J.; Zarghooni, S.; Bschleipfer, T.; Schukowski, K.; Weidner, W.; Wessler, I.; Schwantes, U.; Koepsell, H.; Kummer, W. Acetylcholine and Molecular Components of Its Synthesis and Release Machinery in the Urothelium. Eur. Urol. 2007, 51 (4), 1042-1053.

(62) Cheng, K.; Khurana, S.; Chen, Y.; Kennedy, R. H.; Zimniak, P.; Raufman, J.-P. Lithocholylcholine, a Bile Acid/Acetylcholine Hybrid, Is a Muscarinic Receptor Antagonist. J. Pharmacol. Exp. Ther. 2002, 303 (1), 29-35. 